\title{
Strontium-Catalyzed Highly Enantioselective Michael Additions of Malonates to Enones
}

\author{
Magno Agostinho, and Shū Kobayashi* \\ Department of Chemistry, School of Science and Graduate School of Pharmaceutical \\ Sciences, The University of Tokyo, The HFRE Division, ERATO, JST, Hongo, Bunkyo-ku, \\ Tokyo 113-0033 (Japan)

\section{Supporting Information}

\section{Experimental Section}

\section{General:}

${ }^{1} \mathrm{H}$ and ${ }^{13} \mathrm{C}\left\{{ }^{1} \mathrm{H}\right\}$ NMR spectra were recorded on a JEOL ECX-500 and ECX-600 spectrometer in $\mathrm{CDCl}_{3}$ unless otherwise stated. Tetramethylsilane (TMS) served as internal standard $(\delta=0)$ for ${ }^{1} \mathrm{H}$ and ${ }^{13} \mathrm{C}\left\{{ }^{1} \mathrm{H}\right\}$ NMR. IR spectra were measured with JASCO FT/IR-610 spectrometers. High-performance liquid chromatography was carried out using following apparatuses; SHIMADZU LC-8A, LC-10ATvp, LC-20AB (liquid chromatograph), SHIMADZU SPD-10A (UV detector), SHIMADZU SPD-20A (UV/VIS detector), SHIMADZU SPD-M20A (diode array detector), and SHIMADZU C-R8A (Chromatopac). High resolution mass spectrometry was carried out using BRUKER DALTONICS BioTOF II and JEOL JMS-700. Column chromatography was conducted on Silica gel 60 (Merck), and preparative thin-layer chromatography was carried out using Wakogel B-5F. Calcium bis-iso-propoxide $\left(\mathrm{Ca}(\mathrm{O}-i-\mathrm{Pr})_{2}\right)$ was purchased from Sigma-Aldrich. Strontium bis-iso-propoxide $\left(\mathrm{Sr}(\mathrm{O}-i-\mathrm{Pr})_{2}\right)$ and Barium bis-tert-butoxide $\left(\mathrm{Ba}(\mathrm{O}-t-\mathrm{Bu})_{2}\right)$ were purchased from Kojyundo Kagaku Co., Ltd.. Magnesium bis-tertbutoxide $\left(\mathrm{Mg}(\mathrm{O}-t-\mathrm{Bu})_{2}\right)$ was purchased from Alfa Aesar. Ligands $\mathbf{I},{ }^{1} \mathbf{I I}^{2}$ and $\mathbf{I I I}{ }^{3}$ were prepared according to literature procedures. Malonates 1a-c, 1e and 1f were purchased from Tokyo Chemical Industry (TCI) and distilled before use, malonate 1d was purchased from Wako and distilled before use. Commercially available $\alpha, \beta$-unsaturated carbonyl compounds $2 \mathbf{a}-\mathbf{q}, \mathbf{2 t}$ and $\mathbf{2} \mathbf{u}$ were purchased from TCI, Sigma-Aldrich, Alfa Aesar, Acros or Wako and used as received. Compound $\mathbf{2} \mathbf{r}$ was prepared according to a general procedure reported by Chakraborti ${ }^{4}$ and $2 \mathrm{~s}$ was prepared according to a literature 
method. ${ }^{5}$ Molecular sieves (powder) were purchased from Aldrich and were used after activation $\left(200{ }^{\circ} \mathrm{C},<1 \mathrm{mmHg}, 16 \mathrm{~h}\right)$. Toluene was purchased from Wako as a dry solvent, and distilled in the presence of benzophenone and sodium.

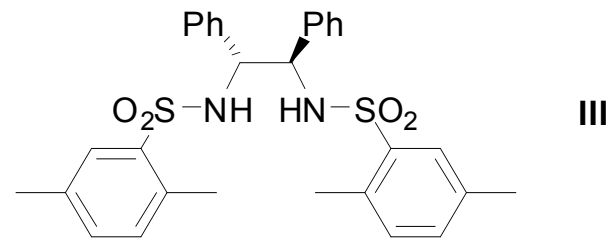

(1R, 2R)-1,2-Diphenylethylene-1,2-bis(2,5-dimethylphenyl)sulfonamide: Prepared according to the procedure reported by Evans. ${ }^{3}{ }^{1} \mathrm{H}$ NMR (600.2 MHz, THF-D 8 , TMS): $\delta$ $=7.37(\mathrm{~s}, 2 \mathrm{H} ; \mathrm{Ar}), 7.06-6.81(\mathrm{~m}, 12 \mathrm{H} ; \mathrm{Ar}, \mathrm{NH}), 6.72-6.67$ (m, 4H; $\mathrm{Ar}), 4.43(\mathrm{~m}, 2 \mathrm{H}, \mathrm{CH})$, $2.40\left(\mathrm{~s}, 6 \mathrm{H}, \mathrm{CH}_{3}\right), 2.14\left(\mathrm{~s}, 6 \mathrm{H}, \mathrm{CH}_{3}\right) ;{ }^{13} \mathrm{C}\left\{{ }^{1} \mathrm{H}\right\}$ NMR $\left(150.9 \mathrm{MHz}, \mathrm{THF}-\mathrm{D}_{8}, \mathrm{TMS}\right): \delta=$ 140.0, 138.2, 136.1, 134.6, 133.2, 132.7, 130.3, 128.7, 128.2, 127.9, 63.3, 20.6, 19.7; $[\alpha]^{21}{ }_{\mathrm{D}}=+40.27\left(c=1.0\right.$ in $\left.\mathrm{CHCl}_{3}\right)$, for the $S S$ enantiomer of III Evans reported ${ }^{3}[\alpha]_{\mathrm{D}}=-$ $42\left(c=0.96\right.$ in $\left.\mathrm{CHCl}_{3}\right)$.

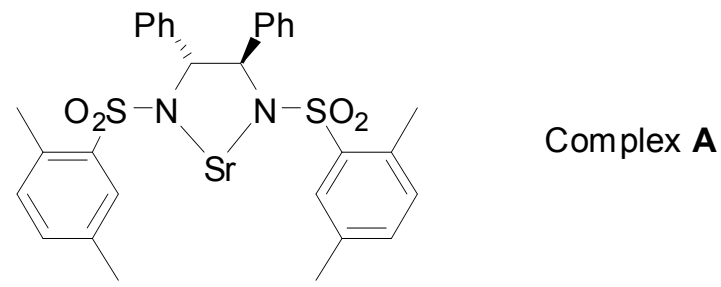

Complex A was prepared by stirring $\operatorname{Sr}(\mathrm{O}-i-\operatorname{Pr})_{2}(0.15 \mathrm{mmol})$ with 1 equivalent of ligand III in deuterated THF $(0.75 \mathrm{~mL})$ for $2 \mathrm{~h}$, it was observed in solution by NMR spectroscopy, but not isolated. At room temperature the peaks corresponding to complex A are significantly broadened, indicating a possible conformational equilibrium; the low solubility of the complex prevented NMR data collection at lower temperatures. The peaks of the free $i$-Pr OH are visible both in the ${ }^{1} \mathrm{H}$ and ${ }^{13} \mathrm{C}\left\{{ }^{1} \mathrm{H}\right\}$ NMR spectrum (see below) and match the values of found for $\mathrm{i}-\mathrm{PrOH}$ in the same solvent. In the ${ }^{13} \mathrm{C}\left\{{ }^{1} \mathrm{H}\right\}$ NMR the NCH carbon atoms from the ligand resonate at $\delta 69.4 \mathrm{ppm}(63.3$ for the free ligand), thus confirming the ligand coordination. ${ }^{1} \mathrm{H}$ NMR (600.2 MHz, THF-D 8 , TMS): $\delta=7.40-6.60$ (br m, 16H; Ar), 4.40 (br m, 2H; CH), 3.86-3.79 (m, 2H; CH free i-PrOH), $3.43\left(\mathrm{~d},{ }^{3} \mathrm{~J}_{\mathrm{HH}}=3.6 \mathrm{~Hz}, 2 \mathrm{H}\right.$; OH free i-PrOH), 2.53 (br s, $6 \mathrm{H} ; \mathrm{CH}_{3}$ ), 1.44 (br s, $6 \mathrm{H} ; \mathrm{CH}_{3}$ ), 
$1.06\left(\mathrm{~d}, J_{\mathrm{HH}}=6.1 \mathrm{~Hz}, 12 \mathrm{H} ; \mathrm{CH}_{3}\right.$ free i-PrOH $) ;{ }^{13} \mathrm{C}\left\{{ }^{1} \mathrm{H}\right\}$ NMR $(150.9 \mathrm{MHz}$, THF-D 8 , TMS): $\delta=145.6,144.8,134.9,134.2,131.6,130.8,129.7,127.7,125.9,69.4,63.6$ (free i-PrOH), 25.9 (free i-PrOH), 21.1, 20.3.

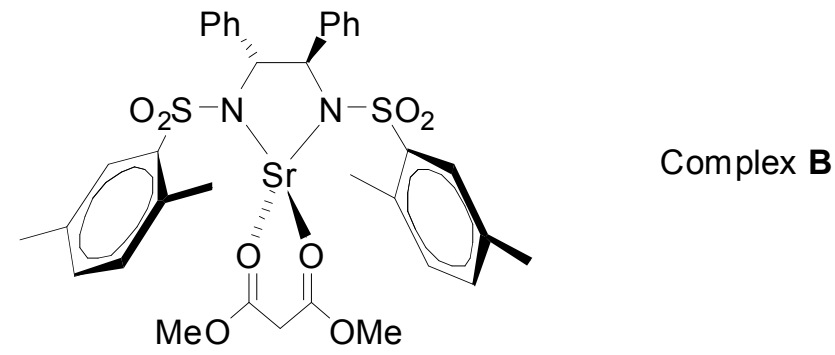

Complex $\mathbf{B}$ was prepared adding 1 equivalent of dimethyl malonate $(\mathbf{1 a}, 17.1 \mu \mathrm{L})$ to the deuterated THF containing complex B. It was observed in solution by NMR spectroscopy, but not isolated. ${ }^{13} \mathrm{C}\left\{{ }^{1} \mathrm{H}\right\}$ NMR (150.9 MHz, THF-D 8 , TMS) selected data: $\delta=174.6$ (COO, coordinated malonate), $68.8\left(\mathrm{NCH}\right.$, ligand), $64.6\left(\mathrm{CH}_{2}\right.$, malonate), 63.6 (free i$\mathrm{PrOH}), 49.7\left(\mathrm{OCH}_{3}\right.$, malonate), 25.9 (free i-PrOH), $20.6\left(\mathrm{CH}_{3}\right.$, ligand), $19.8\left(\mathrm{CH}_{3}\right.$, ligand). For comparison purposes, values of deprotonated dimethyl malonate $\left[\mathrm{CH}^{-}\right.$ $\left.\left(\mathrm{CO}_{2} \mathrm{CH}_{3}\right)_{2}\right]$ in a related ${ }^{6} \mathrm{Sr}$ complex: ${ }^{13} \mathrm{C}\left\{{ }^{1} \mathrm{H}\right\}$ NMR $\left(\mathrm{CDCl}_{3}\right) \delta=173.3(\mathrm{COO}), 62.4$ (CH), $49.7\left(\mathrm{OCH}_{3}\right)$.

\section{Prepared substrates:}

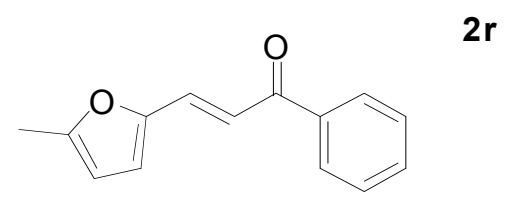

3-(5-Methylfuran-2-yl)-1-phenylprop-2-en-1-one: Prepared according to a general procedure reported by Chakraborti ${ }^{4}$ in $63 \%$ yield. Yellow solid, Mp 57-61 ${ }^{\circ} \mathrm{C}$; IR $\left[\mathrm{cm}^{-1}\right.$ ] (KBr): 1580, 1520, 1367, 1016; ${ }^{1} \mathrm{H}$ NMR (600.2 MHz, $\mathrm{CDCl}_{3}$, TMS): $\delta=8.05-8.01$ (m, $2 \mathrm{H}), 7.58-7.46(\mathrm{~m}, 4 \mathrm{H}), 7.38\left(\mathrm{~d}, J_{\mathrm{HH}}=15.2 \mathrm{~Hz}, 1 \mathrm{H}\right), 6.62\left(\mathrm{~d}, J_{\mathrm{HH}}=3.4 \mathrm{~Hz}, 1 \mathrm{H}\right), 6.13(\mathrm{~d}$, $\left.J_{\mathrm{HH}}=3.4 \mathrm{~Hz}, 1 \mathrm{H}\right), 2.39(\mathrm{~s}, 3 \mathrm{H}) ;{ }^{13} \mathrm{C}\left\{{ }^{1} \mathrm{H}\right\} \mathrm{NMR}\left(150.9 \mathrm{MHz}, \mathrm{CDCl}_{3}, \mathrm{TMS}\right): \delta=189.8$, $155.9,150.3,138.4,132.5,130.8,128.5,128.4,118.3,117.5,109.4,109.3,14.0$; ESIHRMS (m/z) calcd. for $\mathrm{C}_{14} \mathrm{H}_{13} \mathrm{O}_{2}\left((\mathrm{M}+\mathrm{H})^{+}\right): 213.0916$, found: 213.0924 , calcd. for $\mathrm{C}_{14} \mathrm{H}_{12} \mathrm{O}_{2} \mathrm{Na}\left((\mathrm{M}+\mathrm{Na})^{+}\right): 235.0735$, found: 235.0732 . 


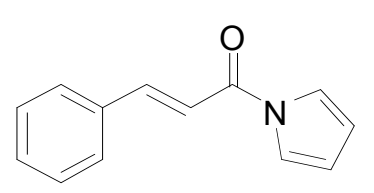

3-Phenyl-1-(1H-pyrrol-1-yl)prop-2-en-1-one: Prepared according to the literature. $^{5}$ White solid, Mp 101-105 ${ }^{\circ} \mathrm{C}$; IR [ $\mathrm{cm}^{-1}$ ] (KBr): 1689, 1624, 1468, 1352; ${ }^{1} \mathrm{H}$ NMR (495.1 $\left.\mathrm{MHz}, \mathrm{CDCl}_{3}, \mathrm{TMS}\right): \delta=7.99\left(\mathrm{~d}, J_{\mathrm{HH}}=15.5 \mathrm{~Hz}, 1 \mathrm{H}\right), 7.64-7.60(\mathrm{~m}, 2 \mathrm{H}), 7.48-7.41(\mathrm{~m}$, $5 \mathrm{H}), 7.14\left(\mathrm{~d}, J_{\mathrm{HH}}=15.5 \mathrm{~Hz}, 1 \mathrm{H}\right), 6.36\left(\right.$ appearance of $\left.\mathrm{t}, J_{\mathrm{HH}}=2.4 \mathrm{~Hz}, 2 \mathrm{H}\right) ;{ }^{13} \mathrm{C}\left\{{ }^{1} \mathrm{H}\right\}$ NMR (150.9 MHz, $\mathrm{CDCl}_{3}$, TMS): $\delta=162.9,147.5,134.2,130.9,129.0,128.4,119.3$, 115.7, 113.4.

Typical experimental procedure for catalytic asymmetric Michael addition reaction reactions of 1a-f to $\alpha, \beta$-unsaturated carbonyl compounds: A typical experimental procedure is described for the reaction of chalcone with diethyl malonate. In a flame dried $30 \mathrm{~mL}$ flask, a suspension of $\operatorname{Sr}(\mathrm{O}-i-\mathrm{Pr})_{2}(0.015 \mathrm{mmol})$, ligand III $(0.018 \mathrm{mmol})$ and MS 4A $(100 \mathrm{mg})$ in toluene $(1.0 \mathrm{~mL})$ was stirred for 2 hours at room temperature. After this period, diethyl malonate $(0.36 \mathrm{mmol})$ in toluene $(1.0 \mathrm{~mL})$ and chalcone $(0.30$ $\mathrm{mmol})$ in toluene $(1.0 \mathrm{~mL})$ were successively added. The reaction was quenched after completion (TLC analysis) by addition of saturated aqueous $\mathrm{NH}_{4} \mathrm{Cl}(10 \mathrm{~mL})$. After addition of dichloromethane $\left(\mathrm{CH}_{2} \mathrm{Cl}_{2}, 10 \mathrm{~mL}\right)$, the organic layer was separated and the aqueous layer extracted three times with $\mathrm{CH}_{2} \mathrm{Cl}_{2}(15 \mathrm{~mL} \times 3)$. The organic layers were combined and dried over anhydrous $\mathrm{Na}_{2} \mathrm{SO}_{4}$. After filtration and concentration under reduced pressure, the crude product was purified by preparative thin layer chromatography (hexane/ethyl acetate $=4 / 1$ ) to afford the desired product. The enantioselectivity was determined by HPLC analysis of the product (see the following analytical data).

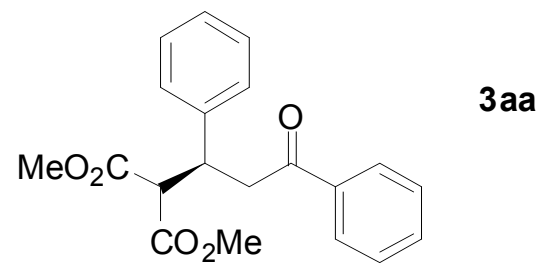


Dimethyl 2-(3-oxo-1,3-diphenylpropyl)malonate ${ }^{7}$ (table 2, entry 1): Prepared according to the typical procedure, as described above in $65 \%$ yield. White solid, $\mathrm{Mp} 77-80{ }^{\circ} \mathrm{C}$; IR $\left[\mathrm{cm}^{-1}\right]$ (KBr): 1730, 1680, 1239, 1157; ${ }^{1} \mathrm{H}$ NMR (600.2 MHz, $\left.\mathrm{CDCl}_{3}, \mathrm{TMS}\right): \delta=7.91$ 7.88 (m, 2H; Ar), 7.55-7.50 (m, 1H; Ar), 7.44-7.39 (m, 2H; Ar), 7.27-7.22 (m, 4H; Ar), 7.20-7.15 (m, 1H; Ar), 4.22-4.16 (m, 1H; CH), 3.88-3.85 (m, $1 \mathrm{H} ; \mathrm{CH}), 3.72\left(\mathrm{~s}, 3 \mathrm{H}, \mathrm{CH}_{3}\right)$, 3.56-3.46 (m, 5H; $\left.\mathrm{CH}_{2}, \mathrm{CH}_{3}\right),{ }^{13} \mathrm{C}\left\{{ }^{1} \mathrm{H}\right\}$ NMR (150.9 MHz, $\left.\mathrm{CDCl}_{3}, \mathrm{TMS}\right): \delta=197.5$, $168.7,168.1,140.4,136.8,133.1,128.5,128.5,128.1$ (from intensity corresponds to 2 peaks), 127.2, 57.3, 52.6, 42.3, 40.7; HPLC (Chiralpak AS-H, hexane $i$-propanol $=19 / 1$, flow rate $0.5 \mathrm{~mL} / \mathrm{min}, \lambda=254 \mathrm{~nm}$ ): $\mathrm{t}_{\text {major }}=38.7 \mathrm{~min}, \mathrm{t}_{\text {minor }}=46.3 \mathrm{~min}$, ee $=94 \% ;[\alpha]_{\mathrm{D}}^{21}=$ $+27.37\left(c=2.0\right.$ in $\left.\mathrm{CHCl}_{3}\right)$, literature value reported by Shibasaki ${ }^{7}$ for the $S$ enantiomer $[\alpha]_{D}^{24}=+25.64\left(c=2.0\right.$ in $\mathrm{CHCl}_{3}, 77 \%$ ee $)$; ESI-HRMS $(\mathrm{m} / \mathrm{z})$ calcd. for $\mathrm{C}_{20} \mathrm{H}_{20} \mathrm{O}_{5} \mathrm{Na}$ $\left((\mathrm{M}+\mathrm{Na})^{+}\right): 363.1208$, found: 363.1282 .

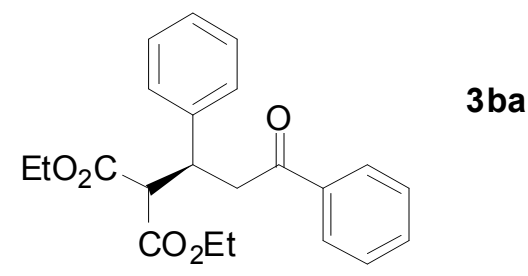

Diethyl 2-(3-oxo-1,3-diphenylpropyl)malonate ${ }^{8,9}$ (table 2, entry 2): Prepared according to the typical procedure, as described above in $97 \%$ yield. White solid, Mp $62-66{ }^{\circ} \mathrm{C}$; IR $\left[\mathrm{cm}^{-1}\right]$ (KBr): 1731, 1685, 1288, 1241; ${ }^{1} \mathrm{H}$ NMR (600.2 MHz, $\left.\mathrm{CDCl}_{3}, \mathrm{TMS}\right): \delta=7.90-$ 7.86 (m, 2H; Ar), 7.52-7.49 (m, 1H; Ar), 7.42-7.38 (m, 2H; Ar), 7.28-7.21 (m, 4H; Ar), 7.17-7.13 (m, 1H; Ar), 4.24-4.14 (m, $\left.3 \mathrm{H} ; \mathrm{CH}, \mathrm{OCH}_{2}\right), 3.94\left(\mathrm{q},{ }^{3} \mathrm{~J}_{\mathrm{HH}}=7.1 \mathrm{~Hz}, 2 \mathrm{H}\right.$; $\left.\mathrm{OCH}_{2}\right), 3.83\left(\mathrm{~d},{ }^{3} J_{\mathrm{HH}}=9.6 \mathrm{~Hz}, 1 \mathrm{H} ; \mathrm{CH}\right), \mathrm{ABM}$ spin system $\left(\mathrm{A}=\mathrm{B}=\mathrm{M}=\mathrm{H}, \delta_{\mathrm{A}}=3.54\right.$, $\left.\delta_{\mathrm{B}}=3.46,{ }^{2} J_{\mathrm{AB}}=16.6,{ }^{3} J_{\mathrm{AM}}=4.4,{ }^{3} J_{\mathrm{BM}}=9.1 \mathrm{~Hz}, 2 \mathrm{H} ; \mathrm{CH}_{2}\right), 1.23\left(\mathrm{t},{ }^{3} J_{\mathrm{HH}}=7.1 \mathrm{~Hz}, 3 \mathrm{H}\right.$; $\left.\mathrm{CH}_{3}\right), 1.00\left(\mathrm{t},{ }^{3} J_{\mathrm{HH}}=7.1 \mathrm{~Hz}, 3 \mathrm{H} ; \mathrm{CH}_{3}\right) ;{ }^{13} \mathrm{C}\left\{{ }^{1} \mathrm{H}\right\} \mathrm{NMR}\left(150.9 \mathrm{MHz}, \mathrm{CDCl}_{3}, \mathrm{TMS}\right): \delta=$ 197.5, 168.4, 167.1, 140.5, 136.8, 133.0, 128.5, 128.4, 128.2, 128.1, 127.1, 61.6, 61.3, 57.6, 42.6, 40.8, 14.0, 13.8; HPLC (Chiralpak AS-H, hexane $/ i$-propanol $=19 / 1$, flow rate $0.5 \mathrm{~mL} / \mathrm{min}, \lambda=254 \mathrm{~nm}$ ): $\mathrm{t}_{\text {major }}=28.1 \mathrm{~min}, \mathrm{t}_{\text {minor }}=31.8 \mathrm{~min}$, ee $=97 \% ;[\alpha]_{\mathrm{D}}^{22}=+19.39$ ( $c=1.0$ in $\left.\mathrm{CHCl}_{3}\right),[\alpha]^{19}{ }_{\mathrm{D}}=+6.35(c=2.5$ in benzene), literature value reported by $\mathrm{Koga}^{9}$ for the $S$ enantiomer $[\alpha]^{25}=+5.4(c=2.61$ in benzene, $82 \%$ ee); ESI-HRMS $(\mathrm{m} / \mathrm{z})$ calcd. for $\mathrm{C}_{22} \mathrm{H}_{24} \mathrm{O}_{5} \mathrm{Na}\left((\mathrm{M}+\mathrm{Na})^{+}\right)$: 391.1521, found: 391.1502 . 


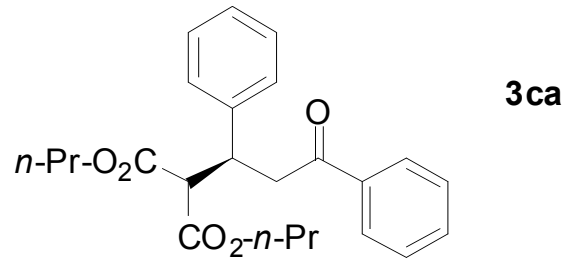

Dipropyl 2-(3-oxo-1,3-diphenylpropyl)malonate (table 2, entry 3): Prepared according to the typical procedure, as described above in $92 \%$ yield. White solid, Mp $55-58{ }^{\circ} \mathrm{C}$; IR $\left[\mathrm{cm}^{-1}\right](\mathrm{KBr}): 1725,1686,1293,1241,1168 ;{ }^{1} \mathrm{H}$ NMR $\left(600.2 \mathrm{MHz}, \mathrm{CDCl}_{3}, \mathrm{TMS}\right): \delta=$ 7.89-7.87 (m, 2H; Ar), 7.52-7.49 (m, 1H; Ar), 7.42-7.38 (m, 2H; Ar), 7.28-7.21 (m, 4H; Ar), 7.17-7.13 (m, 1H; Ar), $4.19\left(\mathrm{t} \mathrm{d},{ }^{3} J_{\mathrm{HH}}=4.5,{ }^{3} J_{\mathrm{HH}}=9.4 \mathrm{~Hz}, 1 \mathrm{H} ; \mathrm{CH}\right), 4.14-4.05(\mathrm{~m}$, $\left.2 \mathrm{H} ; \mathrm{OCH}_{2}\right), 3.87-3.83\left(\mathrm{~m}, 3 \mathrm{H} ; \mathrm{CH}, \mathrm{OCH}_{2}\right), \mathrm{ABM}$ spin system $\left(\mathrm{A}=\mathrm{B}=\mathrm{M}=\mathrm{H}, \delta_{\mathrm{A}}=3.54\right.$, $\delta_{\mathrm{B}}=3.47,{ }^{2} J_{\mathrm{AB}}=16.7,{ }^{3} J_{\mathrm{AM}}=4.6,{ }^{3} J_{\mathrm{BM}}=9.1 \mathrm{~Hz}, 2 \mathrm{H} ; \mathrm{CH}_{2}$ ), 1.63 (appearance of sext, $\left.{ }^{3} J_{\mathrm{HH}}=7.1 \mathrm{~Hz}, 2 \mathrm{H} ; \mathrm{CH}_{2} \mathrm{CH}_{3}\right), 1.47-1.37\left(\mathrm{~m}, 2 \mathrm{H} ; \mathrm{CH}_{2} \mathrm{CH}_{3}\right), 0.90\left(\mathrm{t},{ }^{3} J_{\mathrm{HH}}=7.3 \mathrm{~Hz}, 3 \mathrm{H}\right.$; $\left.\mathrm{CH}_{3}\right), 0.77\left(\mathrm{t},{ }^{3} J_{\mathrm{HH}}=7.5 \mathrm{~Hz}, 3 \mathrm{H} ; \mathrm{CH}_{3}\right) ;{ }^{13} \mathrm{C}\left\{{ }^{1} \mathrm{H}\right\}$ NMR (150.9 MHz, $\left.\mathrm{CDCl}_{3}, \mathrm{TMS}\right): \delta=$ $197.6,168.5,167.9,140.6,136.9,133.0,128.5,128.4,128.2,128.1,127.1,67.2,66.9$, 57.6, 42.6, 40.8, 21.8, 21.6, 10.3, 10.2; HPLC (Chiralpak AS-H, hexane/ $i$-propanol = $100 / 1$, flow rate $0.5 \mathrm{~mL} / \mathrm{min}, \lambda=254 \mathrm{~nm}$ ): $\mathrm{t}_{\text {major }}=47.2 \mathrm{~min}, \mathrm{t}_{\text {minor }}=52.0 \mathrm{~min}$, ee $=99 \%$; $[\alpha]^{21}{ }_{D}=+24.29\left(c=1.0\right.$ in $\left.\mathrm{CHCl}_{3}\right)$; ESI-HRMS $(\mathrm{m} / \mathrm{z})$ calcd. for $\mathrm{C}_{24} \mathrm{H}_{28} \mathrm{O}_{5} \mathrm{Na}\left((\mathrm{M}+\mathrm{Na})^{+}\right)$: 419.1834, found: 419.1865 .

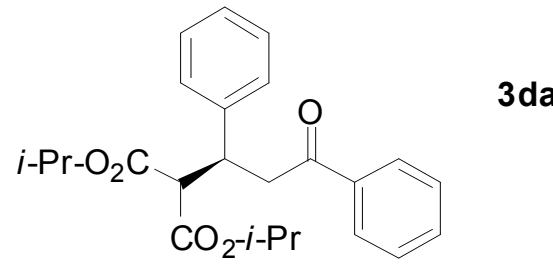

Diisopropyl 2-(3-oxo-1,3-diphenylpropyl)malonate ${ }^{10,11}$ (table 2, entry 7): Prepared according to the typical procedure, as described above in $83 \%$ yield. White solid, Mp 69$71{ }^{\circ} \mathrm{C}$; IR $\left[\mathrm{cm}^{-1}\right](\mathrm{KBr}): 1725,1685,1283,1239,1106 ;{ }^{1} \mathrm{H}$ NMR $\left(600.2 \mathrm{MHz}, \mathrm{CDCl}_{3}\right.$, TMS): $\delta=7.90-7.86(\mathrm{~m}, 2 \mathrm{H} ; \mathrm{Ar}), 7.52-7.48\left(\mathrm{~m}, 1 \mathrm{H}\right.$; Ar), 7.40 (br t, ${ }^{3} J_{\mathrm{HH}}=7.7 \mathrm{~Hz}, 2 \mathrm{H}$; Ar), 7.27-7.19 (m, 4H; Ar), 7.16-7.12 (m, 1H; Ar), 5.07 (sept, $\left.{ }^{3} J_{\mathrm{HH}}=6.2 \mathrm{~Hz}, 1 \mathrm{H} ; \mathrm{CH}\right)$, $4.79\left(\mathrm{sept},{ }^{3} J_{\mathrm{HH}}=6.3 \mathrm{~Hz}, 1 \mathrm{H} ; \mathrm{CH}\right), 4.16\left(\mathrm{t} \mathrm{d},{ }^{3} J_{\mathrm{HH}}=9.7,{ }^{3} J_{\mathrm{HH}}=4.1 \mathrm{~Hz}, 1 \mathrm{H} ; \mathrm{CH}\right), 3.78(\mathrm{~d}$, $\left.{ }^{3} J_{\mathrm{HH}}=9.7 \mathrm{~Hz}, 1 \mathrm{H} ; \mathrm{CH}\right), \mathrm{ABM} \operatorname{spin} \operatorname{system}\left(\mathrm{A}=\mathrm{B}=\mathrm{M}=\mathrm{H}, \delta_{\mathrm{A}}=3.53, \delta_{\mathrm{B}}=3.43,{ }^{2} J_{\mathrm{AB}}=\right.$ 
$\left.16.5,{ }^{3} J_{\mathrm{AM}}=4.1,{ }^{3} J_{\mathrm{BM}}=9.7 \mathrm{~Hz}, 2 \mathrm{H} ; \mathrm{CH}_{2}\right), 1.23\left(\mathrm{~d},{ }^{3} J_{\mathrm{HH}}=6.3 \mathrm{~Hz}, 6 \mathrm{H} ; \mathrm{CH}_{3}\right), 1.04\left(\mathrm{~d},{ }^{3} J_{\mathrm{HH}}\right.$ $\left.=6.2 \mathrm{~Hz}, 3 \mathrm{H} ; \mathrm{CH}_{3}\right), 0.96\left(\mathrm{~d},{ }^{3} J_{\mathrm{HH}}=6.2 \mathrm{~Hz}, 3 \mathrm{H} ; \mathrm{CH}_{3}\right) ;{ }^{13} \mathrm{C}\left\{{ }^{1} \mathrm{H}\right\} \mathrm{NMR}(150.9 \mathrm{MHz}$, $\left.\mathrm{CDCl}_{3}, \mathrm{TMS}\right): \delta=197.6,167.9,167.2,140.5,136.9,133.0,128.5,128.4,128.3,128.1$, 127.0, 69.2, 68.8, 57.9, 42.9, 40.7, 21.7, 21.5, 21.3, 21.3; HPLC (Chiracel OD-H, hexane $/ i$-propanol $=9 / 1$, flow rate $0.5 \mathrm{~mL} / \mathrm{min}, \lambda=254 \mathrm{~nm}$ ): $\mathrm{t}_{\text {major }}=12.4 \mathrm{~min}, \mathrm{t}_{\text {minor }}=$ $13.7 \mathrm{~min}$, ee $=89 \%$; $[\alpha]_{\mathrm{D}}^{22}=+21.27\left(c=1.0\right.$ in $\left.\mathrm{CHCl}_{3}\right)$; ESI-HRMS $(\mathrm{m} / \mathrm{z})$ calcd. for $\mathrm{C}_{24} \mathrm{H}_{28} \mathrm{O}_{5} \mathrm{Na}\left((\mathrm{M}+\mathrm{Na})^{+}\right): 419.1834$, found: 419.1898 .

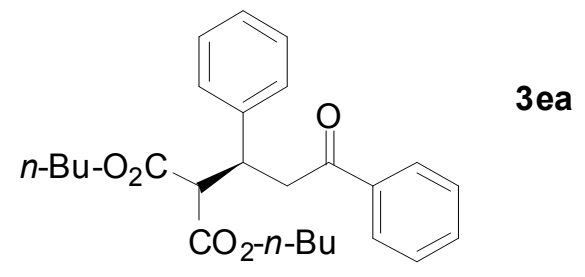

Dibutyl 2-(3-oxo-1,3-diphenylpropyl)malonate ${ }^{8}$ (table 2, entry 8): Prepared according to the typical procedure, as described above in $85 \%$ yield. Colorless oil; IR $\left[\mathrm{cm}^{-1}\right]$ (neat): 1733, 1687, 1254, 1223, 1158; ${ }^{1} \mathrm{H}$ NMR (600.2 MHz, $\mathrm{CDCl}_{3}$, TMS): $\delta=7.90-7.87$ (m, 2H; Ar), 7.52-7.48 (m, 1H; Ar), 7.41-7.38 (m, 2H; Ar), 7.28-7.2o (m, 4H; Ar), 7.17-7.13 (m, 1H; Ar), 4.21-4.09 (m, 3H; CH, $\left.\mathrm{OCH}_{2}\right), 3.92-3.84$ (m, 3H; $\left.\mathrm{CH}, \mathrm{OCH}_{2}\right)$, ABM spin system $\left(\mathrm{A}=\mathrm{B}=\mathrm{M}=\mathrm{H}, \delta_{\mathrm{A}}=3.54, \delta_{\mathrm{B}}=3.46,{ }^{2} J_{\mathrm{AB}}=16.7,{ }^{3} J_{\mathrm{AM}}=4.5,{ }^{3} J_{\mathrm{BM}}=9.3 \mathrm{~Hz}, 2 \mathrm{H}\right.$; $\left.\mathrm{CH}_{2}\right), 1.61-1.55$ (m, 2H; $\left.\mathrm{CH}_{2}\right), 1.41-1.30\left(\mathrm{~m}, 4 \mathrm{H} ; \mathrm{CH}_{2}\right), 1.22-1.15\left(\mathrm{~m}, 2 \mathrm{H} ; \mathrm{CH}_{2}\right), 0.89$ (t, $\left.{ }^{3} J_{\mathrm{HH}}=7.3 \mathrm{~Hz}, 3 \mathrm{H} ; \mathrm{CH}_{3}\right), 0.82\left(\mathrm{t},{ }^{3} J_{\mathrm{HH}}=7.3 \mathrm{~Hz}, 3 \mathrm{H} ; \mathrm{CH}_{3}\right) ;{ }^{13} \mathrm{C}\left\{{ }^{1} \mathrm{H}\right\} \mathrm{NMR}(150.9 \mathrm{MHz}$, $\left.\mathrm{CDCl}_{3}, \mathrm{TMS}\right): \delta=197.5,168.5,167.8,140.6,136.9,133.0,128.5,128.4,128.2,128.1$, 127.1, 65.5, 65.2, 57.6, 42.6, 40.8, 30.5, 30.3, 19.0, 18.9, 13.6, 13.6; HPLC (Chiralpak AS-H, hexane $/ i$-propanol $=100 / 1$, flow rate $1.0 \mathrm{~mL} / \mathrm{min}, \lambda=254 \mathrm{~nm}$ ): $\mathrm{t}_{\text {major }}=28.2 \mathrm{~min}$, $\mathrm{t}_{\text {minor }}=29.9 \mathrm{~min}$, ee $=96 \% ;[\alpha]_{\mathrm{D}}^{22}=+19.65\left(c=1.0\right.$ in $\left.\mathrm{CHCl}_{3}\right)$; ESI-HRMS $(\mathrm{m} / \mathrm{z})$ calcd. for $\mathrm{C}_{26} \mathrm{H}_{32} \mathrm{O}_{5} \mathrm{Na}\left((\mathrm{M}+\mathrm{Na})^{+}\right): 447.2147$, found: 447.2145 , calcd. for $\mathrm{C}_{26} \mathrm{H}_{33} \mathrm{O}_{5}\left((\mathrm{M}+\mathrm{H})^{+}\right)$: 425.2328, found: 425.2316 .

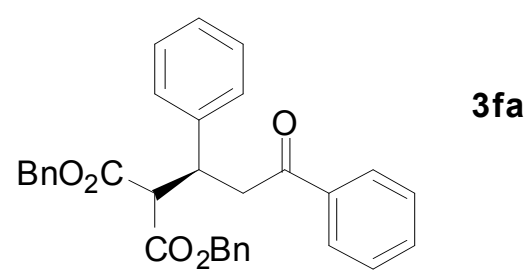


Dibenzyl 2-(3-oxo-1,3-diphenylpropyl)malonate ${ }^{11}$ (table 2, entry 9): Prepared according to the typical procedure, as described above in $85 \%$ yield. White solid, Mp 89$92{ }^{\circ} \mathrm{C}$; IR $\left[\mathrm{cm}^{-1}\right]$ (KBr): 1735, 1682, 1230, 1154; ${ }^{1} \mathrm{H}$ NMR (600.2 MHz, $\left.\mathrm{CDCl}_{3}, \mathrm{TMS}\right): \delta$ = 7.82-7.77 (m, 2H; Ar), 7.52-7.47 (m, 1H; Ar), 7.39-7.35 (m, 2H; Ar), 7.29-7.13 (m, 13H; $\mathrm{Ar}$ ), 7.07-7.04 (m, 2H; $\mathrm{Ar}$ ), 5.18-5.09 (m, $\left.2 \mathrm{H} ; \mathrm{OCH}_{2}\right), 4.90$ (s, $\left.2 \mathrm{H} ; \mathrm{OCH}_{2}\right), 4.25-$ 4.19 (m, 1H; CH), 3.97-3.92 (m, 1H; CH), 3.47-3.42 (m, 2H; $\left.\mathrm{CH}_{2}\right) ;{ }^{13} \mathrm{C}\left\{{ }^{1} \mathrm{H}\right\}$ NMR (150.9 $\left.\mathrm{MHz}, \mathrm{CDCl}_{3}, \mathrm{TMS}\right): \delta=197.3,168.0,167.5,140.3,136.7,135.1,135.0,133.0,128.5$, 128.4, 128.3, 128.1, 128.0, 127.2, 67.3, 67.1, 57.5, 42.2, 40.7; HPLC (Chiralpak AS-H, hexane $/ i$-propanol $=19 / 1$, flow rate $0.5 \mathrm{~mL} / \mathrm{min}, \lambda=254 \mathrm{~nm}$ ): $\mathrm{t}_{\text {major }}=56.3 \mathrm{~min}, \mathrm{t}_{\mathrm{minor}}=$ $63.7 \mathrm{~min}$, ee $=84 \%$; $[\alpha]^{21}{ }_{\mathrm{D}}=+17.55\left(c=1.0\right.$ in $\left.\mathrm{CHCl}_{3}\right)$; ESI-HRMS $(\mathrm{m} / \mathrm{z})$ calcd. for $\mathrm{C}_{32} \mathrm{H}_{28} \mathrm{O}_{5} \mathrm{Na}\left((\mathrm{M}+\mathrm{Na})^{+}\right): 515.1834$, found: 515.1847.

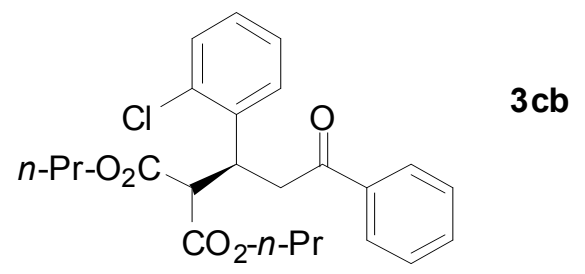

Dipropyl 2-(1-(2-chlorophenyl)-3-oxo-3-phenylpropyl)malonate (table 3, entry 1): Prepared according to the typical procedure, as described above in $76 \%$ yield. Colorless oil; IR $\left[\mathrm{cm}^{-1}\right]$ (neat): 1730, 1687, 1266, 1227, 1160, 738; ${ }^{1} \mathrm{H}$ NMR $\left(600.2 \mathrm{MHz}, \mathrm{CDCl}_{3}\right.$, TMS): $\delta=7.94-7.90(\mathrm{~m}, 2 \mathrm{H} ; \mathrm{Ar}), 7.54-7.49\left(\mathrm{~m}, 1 \mathrm{H} ; \mathrm{Ar}\right.$ ), 7.41 (appearance of br t, ${ }^{3} J_{\mathrm{HH}}=$ 7.8 Hz, 2H; Ar), 7.34-7.30 (m, 2H; Ar), 7.16-7.09 (m, 2H; Ar), $4.66\left(\mathrm{t} \mathrm{d},{ }^{3} \mathrm{~J}_{\mathrm{HH}}=8.8,{ }^{3} \mathrm{JHH}_{\mathrm{HH}}\right.$ $=4.3 \mathrm{~Hz}, 1 \mathrm{H} ; \mathrm{CH}), 4.13-4.00\left(\mathrm{~m}, 3 \mathrm{H} ; \mathrm{CH}, \mathrm{OCH}_{2}\right), 3.94\left(\mathrm{t},{ }^{3} J_{\mathrm{HH}}=6.7 \mathrm{~Hz}, 2 \mathrm{H} ; \mathrm{OCH}_{2}\right)$, $\operatorname{ABM}$ spin system $\left(\mathrm{A}=\mathrm{B}=\mathrm{M}=\mathrm{H}, \delta_{\mathrm{A}}=3.71, \delta_{\mathrm{B}}=3.62,{ }^{2} J_{\mathrm{AB}}=17.2,{ }^{3} J_{\mathrm{AM}}=9.1,{ }^{3} J_{\mathrm{BM}}=\right.$ $\left.4.3 \mathrm{~Hz}, 2 \mathrm{H} ; \mathrm{CH}_{2}\right), 1.63-1.45\left(\mathrm{~m}, 4 \mathrm{H} ; \mathrm{CH}_{2} \mathrm{CH}_{3}\right), 0.86\left(\mathrm{t},{ }^{3} J_{\mathrm{HH}}=7.3 \mathrm{~Hz}, 3 \mathrm{H} ; \mathrm{CH}_{3}\right), 0.83(\mathrm{t}$, $\left.{ }^{3} J_{\mathrm{HH}}=7.5 \mathrm{~Hz}, 3 \mathrm{H} ; \mathrm{CH}_{3}\right) ;{ }^{13} \mathrm{C}\left\{{ }^{1} \mathrm{H}\right\} \mathrm{NMR}\left(150.9 \mathrm{MHz}, \mathrm{CDCl}_{3}, \mathrm{TMS}\right): \delta=197.5,168.4$, 167.9, 137.9, 136.8, 134.1, 133.1, 130.1, 129.4, 128.5, 128.2, 128.1, 126.8, 67.1, 67.1, 55.2, 40.3, 37.4, 21.8, 21.7, 10.2, 10.2; HPLC (Chiracel OJ-H, hexane/i-propanol = 9/1, flow rate $0.5 \mathrm{~mL} / \mathrm{min}, \lambda=254 \mathrm{~nm}$ ): $\mathrm{t}_{\text {major }}=20.5 \mathrm{~min}, \mathrm{t}_{\text {minor }}=26.6 \mathrm{~min}$, ee $=92 \% ;[\alpha]_{\mathrm{D}}^{22}$ $=+41.20\left(c=1.0\right.$ in $\left.\mathrm{CHCl}_{3}\right)$, ESI-HRMS $(\mathrm{m} / \mathrm{z})$ calcd. for $\mathrm{C}_{24} \mathrm{H}_{27} \mathrm{ClO}_{5} \mathrm{Na}\left((\mathrm{M}+\mathrm{Na})^{+}\right)$: 453.1445, found: 453.1437 . 


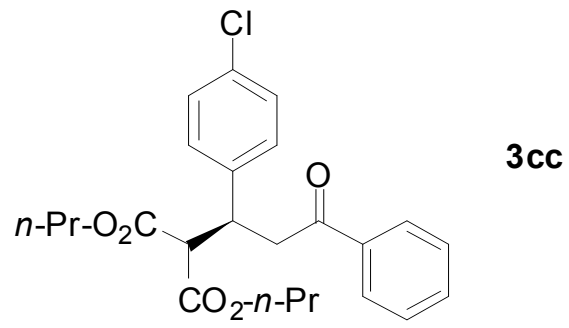

Dipropyl 2-(1-(4-chlorophenyl)-3-oxo-3-phenylpropyl)malonate (table 3, entry 2): Prepared according to the typical procedure, as described above in 93\% yield. White solid, Mp 91-94 ${ }^{\circ} \mathrm{C}$; IR $\left[\mathrm{cm}^{-1}\right]$ (KBr): 1727, 1686, 1239; ${ }^{1} \mathrm{H}$ NMR (600.2 $\mathrm{MHz}, \mathrm{CDCl}_{3}$, TMS): $\delta=7.88\left(\right.$ br d, ${ }^{3} J_{\mathrm{HH}}=7.2 \mathrm{~Hz}, 2 \mathrm{H}$; Ar), 7.52 (appearance of br t, ${ }^{3} J_{\mathrm{HH}}=7.3 \mathrm{~Hz}, 1 \mathrm{H}$; Ar), $7.41\left(\mathrm{t},{ }^{3} \mathrm{~J}_{\mathrm{HH}}=7.8 \mathrm{~Hz}, 2 \mathrm{H}\right.$; Ar), 7.23-7.19 (m, 4H; Ar), 4.19-4.05 (m, 3H; CH, $\left.\mathrm{OCH}_{2}\right), 3.88\left(\mathrm{t},{ }^{3} J_{\mathrm{HH}}=6.6 \mathrm{~Hz}, 2 \mathrm{H} ; \mathrm{OCH}_{2}\right), 3.82\left(\mathrm{~d},{ }^{3} J_{\mathrm{HH}}=9.5 \mathrm{~Hz}, 1 \mathrm{H} ; \mathrm{CH}\right), \mathrm{ABM}$ spin system $\left(\mathrm{A}=\mathrm{B}=\mathrm{M}=\mathrm{H}, \delta_{\mathrm{A}}=3.53, \delta_{\mathrm{B}}=3.45,{ }^{2} J_{\mathrm{AB}}=17.0,{ }^{3} J_{\mathrm{AM}}=4.2,{ }^{3} J_{\mathrm{BM}}=9.5 \mathrm{~Hz}, 2 \mathrm{H}\right.$; $\mathrm{CH}_{2}$ ), 1.64 (appearance of sext, $\left.{ }^{3} \mathrm{~J}_{\mathrm{HH}}=7.2 \mathrm{~Hz}, 2 \mathrm{H} ; \mathrm{CH}_{2} \mathrm{CH}_{3}\right), 1.50-1.41(\mathrm{~m}, 2 \mathrm{H}$; $\left.\mathrm{CH}_{2} \mathrm{CH}_{3}\right), 0.90\left(\mathrm{t},{ }^{3} \mathrm{~J}_{\mathrm{HH}}=7.3 \mathrm{~Hz}, 3 \mathrm{H} ; \mathrm{CH}_{3}\right), 0.79\left(\mathrm{t},{ }^{3} J_{\mathrm{HH}}=7.5 \mathrm{~Hz}, 3 \mathrm{H} ; \mathrm{CH}_{3}\right) ;{ }^{13} \mathrm{C}\left\{{ }^{1} \mathrm{H}\right\}$ NMR (150.9 MHz, $\left.\mathrm{CDCl}_{3}, \mathrm{TMS}\right): \delta=197.3,168.2,167.7,139.1,136.7,133.2,132.9$, $129.7,128.6,128.5,128.0,67.3,67.1,57.4,42.4,40.2,21.8,21.7,10.3,10.2$; HPLC (Chiracel OJ-H, hexane $/ i$-propanol $=19 / 1$, flow rate $0.3 \mathrm{~mL} / \mathrm{min}, \lambda=254 \mathrm{~nm}$ ): $\mathrm{t}_{\text {major }}=$ 47.8 min, $\mathrm{t}_{\text {minor }}=54.8 \mathrm{~min}$, ee $=97 \% ;[\alpha]_{\mathrm{D}}^{22}=+24.09\left(c=1.0\right.$ in $\left.\mathrm{CHCl}_{3}\right)$; ESI-HRMS (m/z) calcd. for $\mathrm{C}_{24} \mathrm{H}_{27} \mathrm{ClO}_{5} \mathrm{Na}\left((\mathrm{M}+\mathrm{Na})^{+}\right)$: 453.1445, found: 453.1486.

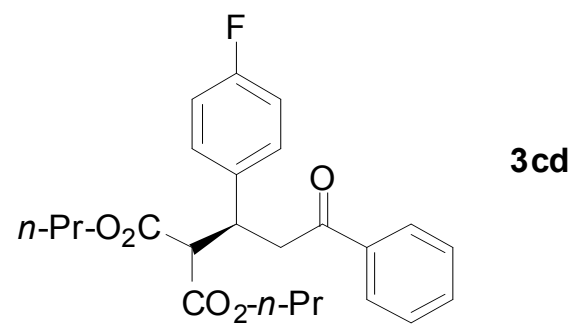

Dipropyl 2-(1-(4-fluorophenyl)-3-oxo-3-phenylpropyl)malonate (table 3, entry 3): Prepared according to the typical procedure, as described above in $92 \%$ yield. White solid, Mp 35-38 ${ }^{\circ} \mathrm{C}$; IR $\left[\mathrm{cm}^{-1}\right]$ (neat): 1733, 1687, 1510, 1226, 1161, 739; ${ }^{1} \mathrm{H}$ NMR (600.2 $\left.\mathrm{MHz}, \mathrm{CDCl}_{3}, \mathrm{TMS}\right): \delta=7.91-7.87$ (m, 2H; Ar), 7.52 (appearance of br t, ${ }^{3} J_{\mathrm{HH}}=7.5 \mathrm{~Hz}$, $1 \mathrm{H} ; \mathrm{Ar}$ ), $7.42\left(\mathrm{t},{ }^{3} J_{\mathrm{HH}}=7.8 \mathrm{~Hz}, 2 \mathrm{H} ; \mathrm{Ar}\right.$ ), 7.27-7.23 (m, 2H; Ar), 6.92 (appearance of br t, $\left.{ }^{3} J_{\mathrm{HH}}=8.7 \mathrm{~Hz}, 2 \mathrm{H} ; \mathrm{Ar}\right), 4.20-4.06\left(\mathrm{~m}, 3 \mathrm{H} ; \mathrm{CH}, \mathrm{OCH}_{2}\right), 3.87\left(\mathrm{t},{ }^{3} J_{\mathrm{HH}}=6.8 \mathrm{~Hz}, 2 \mathrm{H} ; \mathrm{OCH}_{2}\right)$, 
$3.82\left(\mathrm{~d},{ }^{3} J_{\mathrm{HH}}=9.6 \mathrm{~Hz}, 1 \mathrm{H} ; \mathrm{CH}\right), \mathrm{ABM}$ spin system $\left(\mathrm{A}=\mathrm{B}=\mathrm{M}=\mathrm{H}, \delta_{\mathrm{A}}=3.53, \delta_{\mathrm{B}}=3.44\right.$, ${ }^{2} J_{\mathrm{AB}}=16.6,{ }^{3} J_{\mathrm{AM}}=4.3,{ }^{3} J_{\mathrm{BM}}=9.6 \mathrm{~Hz}, 2 \mathrm{H} ; \mathrm{CH}_{2}$ ), 1.64 (appearance of sext, ${ }^{3} J_{\mathrm{HH}}=7.0 \mathrm{~Hz}$, $\left.2 \mathrm{H} ; \mathrm{CH}_{2} \mathrm{CH}_{3}\right), 1.50-1.40\left(\mathrm{~m}, 2 \mathrm{H} ; \mathrm{CH}_{2} \mathrm{CH}_{3}\right), 0.91\left(\mathrm{t},{ }^{3} \mathrm{~J}_{\mathrm{HH}}=7.5 \mathrm{~Hz}, 3 \mathrm{H} ; \mathrm{CH}_{3}\right), 0.79\left(\mathrm{t},{ }^{3} \mathrm{~J}_{\mathrm{HH}}\right.$ $\left.=7.5 \mathrm{~Hz}, 3 \mathrm{H} ; \mathrm{CH}_{3}\right) ;{ }^{13} \mathrm{C}\left\{{ }^{1} \mathrm{H}\right\}$ NMR $\left(150.9 \mathrm{MHz}, \mathrm{CDCl}_{3}, \mathrm{TMS}\right): \delta=197.4,168.3,167.8$, $161.8\left(\mathrm{~d}, J_{\mathrm{CF}}=245.4 \mathrm{~Hz}\right), 136.8,136.3,133.1,129.9\left(\mathrm{~d}, J_{\mathrm{CF}}=7.9 \mathrm{~Hz}\right), 128.6,128.1$, $115.2\left(\mathrm{~d}, J_{\mathrm{CF}}=21.2 \mathrm{~Hz}\right), 67.3,67.0,57.6,42.6,40.1,21.8,21.7,10.3,10.2 ;$ HPLC (Chiracel OJ-H, hexane $/ i$-propanol $=19 / 1$, flow rate $0.3 \mathrm{~mL} / \mathrm{min}, \lambda=254 \mathrm{~nm}$ ): $\mathrm{t}_{\text {major }}=$ 50.7 min, $t_{\text {minor }}=63.1 \mathrm{~min}$, ee $=98 \%$; $[\alpha]^{21}{ }_{\mathrm{D}}=+25.21\left(c=1.0\right.$ in $\left.\mathrm{CHCl}_{3}\right)$; ESI-HRMS $(\mathrm{m} / \mathrm{z})$ calcd. for $\mathrm{C}_{24} \mathrm{H}_{27} \mathrm{FO}_{5} \mathrm{Na}\left((\mathrm{M}+\mathrm{Na})^{+}\right): 437.1740$, found: 437.1728 .

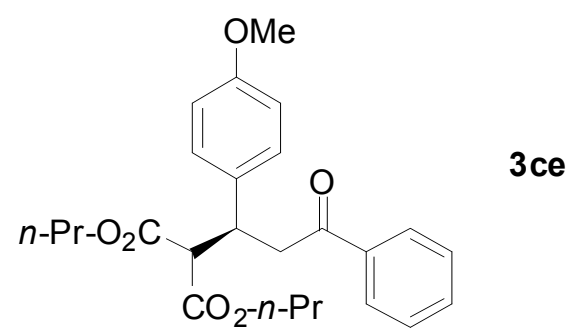

Dipropyl 2-(1-(4-methoxyphenyl)-3-oxo-3-phenylpropyl)malonate (table 3, entry 4): Prepared according to the typical procedure, as described above in $80 \%$ yield. White solid, Mp 41-44 ${ }^{\circ} \mathrm{C}$; IR $\left[\mathrm{cm}^{-1}\right.$ ] (KBr): 1729, 1678, 1511, 1243, 1162; ${ }^{1} \mathrm{H}$ NMR (600.2 $\mathrm{MHz}, \mathrm{CDCl}_{3}$, TMS): $\delta=7.89$ (br d, ${ }^{3} J_{\mathrm{HH}}=7.1 \mathrm{~Hz}, 2 \mathrm{H} ; \mathrm{Ar}$ ), 7.50 (appearance of br t, ${ }^{3} J_{\mathrm{HH}}$ $=7.5 \mathrm{~Hz}, 1 \mathrm{H} ; \mathrm{Ar}), 7.40\left(\mathrm{t},{ }^{3} \mathrm{~J}_{\mathrm{HH}}=7.8 \mathrm{~Hz}, 2 \mathrm{H} ; \mathrm{Ar}\right), 7.17\left(\mathrm{~d},{ }^{3} J_{\mathrm{HH}}=8.7 \mathrm{~Hz}, 2 \mathrm{H} ; \mathrm{Ar}\right), 6.76$ $\left(\mathrm{d},{ }^{3} J_{\mathrm{HH}}=8.5 \mathrm{~Hz}, 2 \mathrm{H} ; \mathrm{Ar}\right), 4.16-4.05\left(\mathrm{~m}, 3 \mathrm{H} ; \mathrm{CH}, \mathrm{OCH}_{2}\right), 3.86\left(\mathrm{t},{ }^{3} J_{\mathrm{HH}}=6.6 \mathrm{~Hz}, 2 \mathrm{H}\right.$; $\left.\mathrm{OCH}_{2}\right), 3.82\left(\mathrm{~d},{ }^{3} J_{\mathrm{HH}}=9.6 \mathrm{~Hz}, 1 \mathrm{H} ; \mathrm{CH}\right), 3.72\left(\mathrm{~s}, 3 \mathrm{H} ; \mathrm{OCH}_{3}\right), \mathrm{ABM}$ spin system $(\mathrm{A}=\mathrm{B}=$ $\left.\mathrm{M}=\mathrm{H}, \delta_{\mathrm{A}}=3.51, \delta_{\mathrm{B}}=3.42,{ }^{2} J_{\mathrm{AB}}=16.5,{ }^{3} J_{\mathrm{AM}}=4.2,{ }^{3} J_{\mathrm{BM}}=9.5 \mathrm{~Hz}, 2 \mathrm{H} ; \mathrm{CH}_{2}\right), 1.64$ (appearance of sext, $\left.{ }^{3} J_{\mathrm{HH}}=7.1 \mathrm{~Hz}, 2 \mathrm{H} ; \mathrm{CH}_{2} \mathrm{CH}_{3}\right), 1.50-1.40\left(\mathrm{~m}, 2 \mathrm{H} ; \mathrm{CH}_{2} \mathrm{CH}_{3}\right), 0.91(\mathrm{t}$, $\left.{ }^{3} J_{\mathrm{HH}}=7.5 \mathrm{~Hz}, 3 \mathrm{H} ; \mathrm{CH}_{3}\right), 0.79\left(\mathrm{t},{ }^{3} J_{\mathrm{HH}}=7.5 \mathrm{~Hz}, 3 \mathrm{H} ; \mathrm{CH}_{3}\right) ;{ }^{13} \mathrm{C}\left\{{ }^{1} \mathrm{H}\right\} \mathrm{NMR}(150.9 \mathrm{MHz}$, $\left.\mathrm{CDCl}_{3}, \mathrm{TMS}\right): \delta=197.7,168.5,167.9,158.6,136.9,133.0,132.5,129.2,128.5,128.1$, 113.8, 67.2, 66.9, 57.8, 55.1, 42.8, 40.2, 21.8, 21.7, 10.3, 10.2; HPLC (Chiracel OJ-H, hexane $/ i$-propanol $=9 / 1$, flow rate $1.5 \mathrm{~mL} / \mathrm{min}, \lambda=254 \mathrm{~nm}$ ): $\mathrm{t}_{\text {major }}=17.6 \mathrm{~min}, \mathrm{t}_{\mathrm{minor}}=$ $11.0 \mathrm{~min}$, ee $>99 \%$; $[\alpha]^{21}{ }_{\mathrm{D}}=+17.51\left(c=1.0\right.$ in $\left.\mathrm{CHCl}_{3}\right)$; ESI-HRMS $(\mathrm{m} / \mathrm{z})$ calcd. for $\mathrm{C}_{25} \mathrm{H}_{30} \mathrm{O}_{6} \mathrm{Na}\left((\mathrm{M}+\mathrm{Na})^{+}\right): 449.1940$, found: 449.1939 . 


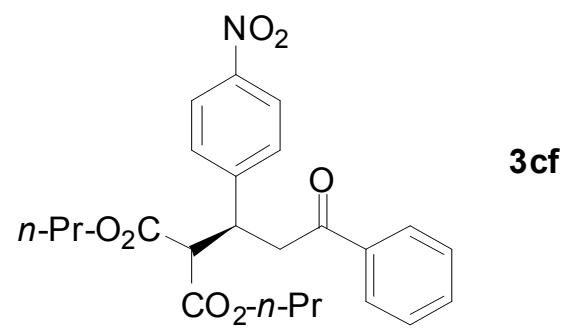

Dipropyl 2-(1-(4-nitrophenyl)-3-oxo-3-phenylpropyl)malonate (table 3, entry 5): Prepared according to the typical procedure, as described above in $98 \%$ yield. White solid, Mp 82-86 ${ }^{\circ} \mathrm{C}$; IR $\left[\mathrm{cm}^{-1}\right]$ (KBr): 1726, 1686, 1519, 1346, 1239, 1151; ${ }^{1} \mathrm{H}$ NMR (600.2 MHz, $\left.\mathrm{CDCl}_{3}, \mathrm{TMS}\right): \delta$ = 8.12-8.09 (m, 2H; Ar), 7.90-7.87 (m, 2H; Ar), 7.56-7.49 (m, 3H; Ar), 7.45-7.41 (m, 2H; Ar), $4.31\left(\mathrm{t} \mathrm{d},{ }^{3} J_{\mathrm{HH}}=4.3,{ }^{3} J_{\mathrm{HH}}=9.4 \mathrm{~Hz}, 1 \mathrm{H} ; \mathrm{CH}\right), 4.17-$ $4.07\left(\mathrm{~m}, 2 \mathrm{H} ; \mathrm{OCH}_{2}\right), 3.93-3.86\left(\mathrm{~m}, 3 \mathrm{H} ; \mathrm{CH}, \mathrm{OCH}_{2}\right), \mathrm{ABM}$ spin system $(\mathrm{A}=\mathrm{B}=\mathrm{M}=\mathrm{H}$, $\delta_{\mathrm{A}}=3.61, \delta_{\mathrm{B}}=3.55,{ }^{2} J_{\mathrm{AB}}=17.3,{ }^{3} J_{\mathrm{AM}}=4.2,{ }^{3} J_{\mathrm{BM}}=9.5 \mathrm{~Hz}, 2 \mathrm{H} ; \mathrm{CH}_{2}$ ), 1.64 (appearance of sext, $\left.{ }^{3} J_{\mathrm{HH}}=7.0 \mathrm{~Hz}, 2 \mathrm{H} ; \mathrm{CH}_{2} \mathrm{CH}_{3}\right), 1.52-1.43\left(\mathrm{~m}, 2 \mathrm{H} ; \mathrm{CH}_{2} \mathrm{CH}_{3}\right), 0.91\left(\mathrm{t},{ }^{3} J_{\mathrm{HH}}=7.3 \mathrm{~Hz}\right.$, $\left.3 \mathrm{H} ; \mathrm{CH}_{3}\right), 0.80\left(\mathrm{t},{ }^{3} \mathrm{JHH}_{\mathrm{HH}}=7.4 \mathrm{~Hz}, 3 \mathrm{H} ; \mathrm{CH}_{3}\right) ;{ }^{13} \mathrm{C}\left\{{ }^{1} \mathrm{H}\right\} \mathrm{NMR}\left(150.9 \mathrm{MHz}, \mathrm{CDCl}_{3}, \mathrm{TMS}\right): \delta$ $=196.8,167.9,167.4,148.6,147.0,136.5,133.4,129.4,128.7,128.0,123.6,67.5,67.3$, 56.9, 42.0, 40.4, 21.8, 21.7, 10.2, 10.2; HPLC (Chiracel OJ-H, hexane/i-propanol = 9/1, flow rate $1.0 \mathrm{~mL} / \mathrm{min}, \lambda=254 \mathrm{~nm}): \mathrm{t}_{\text {major }}=28.5 \mathrm{~min}, \mathrm{t}_{\text {minor }}=41.2 \mathrm{~min}$, ee $=96 \% ;[\alpha]^{22}{ }_{\mathrm{D}}$ $=+31.37\left(c=1.0\right.$ in $\left.\mathrm{CHCl}_{3}\right)$; ESI-HRMS $(\mathrm{m} / \mathrm{z})$ calcd. for $\mathrm{C}_{24} \mathrm{H}_{27} \mathrm{NO}_{7} \mathrm{Na}\left((\mathrm{M}+\mathrm{Na})^{+}\right)$: 464.1685, found: 464.1644 .

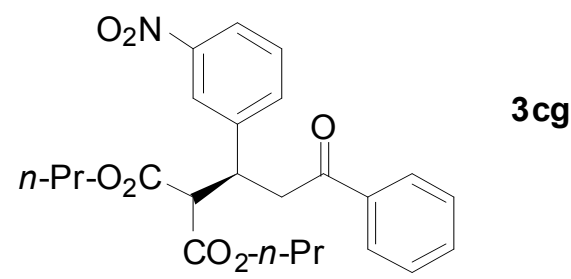

Dipropyl 2-(1-(3-nitrophenyl)-3-oxo-3-phenylpropyl)malonate (table 3, entry 6): Prepared according to the typical procedure, as described above in $94 \%$ yield. Colorless oil; IR $\left[\mathrm{cm}^{-1}\right]$ (neat): 1731, 1686, 1638, 1532, 1352, 1265, 738; ${ }^{1} \mathrm{H}$ NMR $(600.2 \mathrm{MHz}$, $\left.\mathrm{CDCl}_{3}, \mathrm{TMS}\right): \delta=8.18\left(\mathrm{t}, J_{\mathrm{HH}}=1.9 \mathrm{~Hz}, 1 \mathrm{H} ; \mathrm{Ar}\right), 8.04\left(\mathrm{~d} \mathrm{~d}, J_{\mathrm{HH}}=1.8 \mathrm{~Hz}, J_{\mathrm{HH}}=8.2 \mathrm{~Hz}\right.$, $1 \mathrm{H}$; Ar), 7.89 (br d, $\left.J_{\mathrm{HH}}=7.2 \mathrm{~Hz}, 2 \mathrm{H} ; \mathrm{Ar}\right), 7.71$ (br d, $\left.J_{\mathrm{HH}}=7.8 \mathrm{~Hz}, 1 \mathrm{H} ; \mathrm{Ar}\right), 7.54$ ((br t, $\left.J_{\mathrm{HH}}=7.3 \mathrm{~Hz}, 1 \mathrm{H} ; \mathrm{Ar}\right), 7.46-7.41(\mathrm{~m}, 3 \mathrm{H} ; \mathrm{Ar}), 4.32\left(\mathrm{t} \mathrm{d},{ }^{3} J_{\mathrm{HH}}=4.3,{ }^{3} J_{\mathrm{HH}}=9.3 \mathrm{~Hz}, 1 \mathrm{H}\right.$; $\mathrm{CH}), 4.17-4.08\left(\mathrm{~m}, 2 \mathrm{H} ; \mathrm{OCH}_{2}\right), 3.92-3.88\left(\mathrm{~m}, 3 \mathrm{H} ; \mathrm{CH}, \mathrm{OCH}_{2}\right), \mathrm{ABM}$ spin system $(\mathrm{A}=\mathrm{B}$ 
$\left.=\mathrm{M}=\mathrm{H}, \delta_{\mathrm{A}}=3.63, \delta_{\mathrm{B}}=3.57,{ }^{2} J_{\mathrm{AB}}=17.4,{ }^{3} J_{\mathrm{AM}}=4.4,{ }^{3} J_{\mathrm{BM}}=9.4 \mathrm{~Hz}, 2 \mathrm{H} ; \mathrm{CH}_{2}\right), 1.64$ (appearance of sext, ${ }^{3} J_{\mathrm{HH}}=7.1 \mathrm{~Hz}, 2 \mathrm{H} ; \mathrm{CH}_{2} \mathrm{CH}_{3}$ ), 1.52-1.41 (m, 2H; $\left.\mathrm{CH}_{2} \mathrm{CH}_{3}\right), 0.91$ (t, $\left.{ }^{3} J_{\mathrm{HH}}=7.5 \mathrm{~Hz}, 3 \mathrm{H} ; \mathrm{CH}_{3}\right), 0.79\left(\mathrm{t},{ }^{3} J_{\mathrm{HH}}=7.4 \mathrm{~Hz}, 3 \mathrm{H} ; \mathrm{CH}_{3}\right) ;{ }^{13} \mathrm{C}\left\{{ }^{1} \mathrm{H}\right\} \mathrm{NMR}(150.9 \mathrm{MHz}$, $\left.\mathrm{CDCl}_{3}, \mathrm{TMS}\right): \delta=196.9,168.0,167.5,148.2,143.1,136.5,135.3,133.4,129.3,128.7$, 128.0, 123.1, 122.2, 67.5, 67.2, 57.0, 42.1, 40.2, 21.8, 21.7, 10.2, 10.1; HPLC (Chiralpak AD-H, hexane $/ i$-propanol $=9 / 1$, flow rate $1.0 \mathrm{~mL} / \mathrm{min}, \lambda=254 \mathrm{~nm}$ ): $\mathrm{t}_{\text {major }}=48.9 \mathrm{~min}$, $\mathrm{t}_{\text {minor }}=32.1 \mathrm{~min}$, ee $=94 \% ;[\alpha]_{\mathrm{D}}^{23}=+31.81\left(c=1.0\right.$ in $\left.\mathrm{CHCl}_{3}\right)$; ESI-HRMS $(\mathrm{m} / \mathrm{z})$ calcd. for $\mathrm{C}_{24} \mathrm{H}_{27} \mathrm{NO}_{7} \mathrm{Na}\left((\mathrm{M}+\mathrm{Na})^{+}\right): 464.1685$, found: 464.1658 .

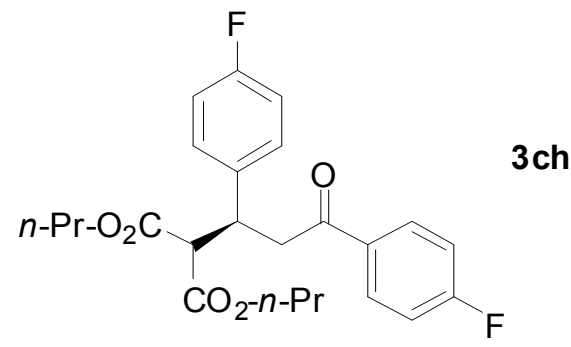

Dipropyl 2-(1,3-bis(4-fluorophenyl)-3-oxopropyl)malonate (table 3, entry 7): Prepared according to the typical procedure, as described above in $91 \%$ yield. Colorless oil; IR $\left[\mathrm{cm}^{-1}\right]$ (neat): 1732, 1686, 1600, 1510, 1228, 1157; ${ }^{1} \mathrm{H}$ NMR (600.2 MHz, $\left.\mathrm{CDCl}_{3}, \mathrm{TMS}\right)$ : $\delta=7.94-7.91(\mathrm{~m}, 2 \mathrm{H} ; \mathrm{Ar}), 7.26-7.22(\mathrm{~m}, 2 \mathrm{H} ; \mathrm{Ar}), 7.11-7.06(\mathrm{~m}, 2 \mathrm{H} ; \mathrm{Ar}), 6.95-6.90(\mathrm{~m}$, $2 \mathrm{H} ; \mathrm{Ar}), 4.18-4.06\left(\mathrm{~m}, 3 \mathrm{H} ; \mathrm{CH}, \mathrm{OCH}_{2}\right), 3.86\left(\mathrm{t},{ }^{3} J_{\mathrm{HH}}=6.6 \mathrm{~Hz}, 2 \mathrm{H} ; \mathrm{OCH}_{2}\right), 3.81\left(\mathrm{~d},{ }^{3} J_{\mathrm{HH}}\right.$ $=9.6 \mathrm{~Hz}, 1 \mathrm{H} ; \mathrm{CH}), 3.72\left(\mathrm{~s}, 3 \mathrm{H} ; \mathrm{OCH}_{3}\right), \mathrm{ABM}$ spin system $\left(\mathrm{A}=\mathrm{B}=\mathrm{M}=\mathrm{H}, \delta_{\mathrm{A}}=3.50, \delta_{\mathrm{B}}\right.$ $=3.38,{ }^{2} J_{\mathrm{AB}}=16.4,{ }^{3} J_{\mathrm{AM}}=4.2,{ }^{3} J_{\mathrm{BM}}=9.6 \mathrm{~Hz}, 2 \mathrm{H} ; \mathrm{CH}_{2}$ ), 1.64 (appearance of sext, ${ }^{3} J_{\mathrm{HH}}=$ $\left.7.1 \mathrm{~Hz}, 2 \mathrm{H} ; \mathrm{CH}_{2} \mathrm{CH}_{3}\right), 1.50-1.40\left(\mathrm{~m}, 2 \mathrm{H} ; \mathrm{CH}_{2} \mathrm{CH}_{3}\right), 0.91\left(\mathrm{t},{ }^{3} J_{\mathrm{HH}}=7.5 \mathrm{~Hz}, 3 \mathrm{H} ; \mathrm{CH}_{3}\right)$, $0.79\left(\mathrm{t},{ }^{3} \mathrm{~J}_{\mathrm{HH}}=7.4 \mathrm{~Hz}, 3 \mathrm{H} ; \mathrm{CH}_{3}\right) ;{ }^{13} \mathrm{C}\left\{{ }^{1} \mathrm{H}\right\}$ NMR $\left(150.9 \mathrm{MHz}, \mathrm{CDCl}_{3}, \mathrm{TMS}\right): \delta=195.9$, $168.4,167.8,165.8\left(\mathrm{~d}, J_{\mathrm{CF}}=254.7 \mathrm{~Hz}\right), 161.7\left(\mathrm{~d}, J_{\mathrm{CF}}=245.7 \mathrm{~Hz}\right), 136.1,133.2,130.8(\mathrm{~d}$, $\left.J_{\mathrm{CF}}=9.0 \mathrm{~Hz}\right), 129.9\left(\mathrm{~d}, J_{\mathrm{CF}}=7.9 \mathrm{~Hz}\right), 115.7\left(\mathrm{~d}, J_{\mathrm{CF}}=21.8 \mathrm{~Hz}\right), 115.3\left(\mathrm{~d}, J_{\mathrm{CF}}=21.3 \mathrm{~Hz}\right)$, 67.3, 67.1, 57.6, 42.6, 40.2, 21.9, 21.7, 10.3, 10.2; HPLC (Chiralpak AS-H, hexane/ipropanol $=9 / 1$, flow rate $0.5 \mathrm{~mL} / \mathrm{min}, \lambda=254 \mathrm{~nm}$ ): $\mathrm{t}_{\text {major }}=20.3 \mathrm{~min}, \mathrm{t}_{\text {minor }}=23.4 \mathrm{~min}$, ee $=96 \% ;[\alpha]^{22}=+21.56\left(c=1.0\right.$ in $\left.\mathrm{CHCl}_{3}\right)$; ESI-HRMS $(\mathrm{m} / \mathrm{z})$ calcd. for $\mathrm{C}_{24} \mathrm{H}_{26} \mathrm{~F}_{2} \mathrm{O}_{5} \mathrm{Na}$ $\left((\mathrm{M}+\mathrm{Na})^{+}\right): 455.1646$, found: 455.1668 . 


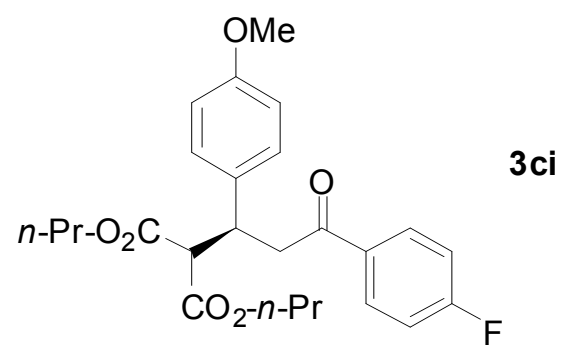

Dipropyl 2-(3-(4-fluorophenyl)-1-(4-methoxyphenyl)-3-oxopropyl)malonate (table 3, entry 8): Prepared according to the typical procedure, as described above in $81 \%$ yield. White solid, Mp 60-63 ${ }^{\circ} \mathrm{C}$; IR [ $\mathrm{cm}^{-1}$ ] (neat): 1731, 1686, 1599, 1514, 1266, 1251, 1157 , 739; ${ }^{1} \mathrm{H}$ NMR (600.2 MHz, $\mathrm{CDCl}_{3}$, TMS): $\delta$ = 7.94-7.91 (m, 2H; Ar), 7.18-7.15 (m, 2H; Ar), 7.07 (appearance of br t, ${ }^{3} J_{\mathrm{HH}}=8.6 \mathrm{~Hz}, 2 \mathrm{H}$; Ar), 6.78-6.75 (m, 2H; Ar), 4.15-4.05 $\left(\mathrm{m}, 3 \mathrm{H} ; \mathrm{CH}, \mathrm{OCH}_{2}\right), 3.87\left(\mathrm{t},{ }^{3} J_{\mathrm{HH}}=6.7 \mathrm{~Hz}, 2 \mathrm{H} ; \mathrm{OCH}_{2}\right), 3.82\left(\mathrm{~d},{ }^{3} J_{\mathrm{HH}}=9.6 \mathrm{~Hz}, 1 \mathrm{H} ; \mathrm{CH}\right)$, $\operatorname{ABM}$ spin system $\left(\mathrm{A}=\mathrm{B}=\mathrm{M}=\mathrm{H}, \delta_{\mathrm{A}}=3.53, \delta_{\mathrm{B}}=3.40,{ }^{2} J_{\mathrm{AB}}=16.7,{ }^{3} J_{\mathrm{AM}}=4.3,{ }^{3} J_{\mathrm{BM}}=\right.$ $\left.9.6 \mathrm{~Hz}, 2 \mathrm{H} ; \mathrm{CH}_{2}\right), 1.68-1.61\left(\mathrm{~m}, 2 \mathrm{H} ; \mathrm{CH}_{2} \mathrm{CH}_{3}\right), 1.50-1.40\left(\mathrm{~m}, 2 \mathrm{H} ; \mathrm{CH}_{2} \mathrm{CH}_{3}\right), 0.91$ (t, ${ }^{3} J_{\mathrm{HH}}$ $\left.=7.5 \mathrm{~Hz}, 3 \mathrm{H} ; \mathrm{CH}_{3}\right), 0.79\left(\mathrm{t},{ }^{3} \mathrm{~J}_{\mathrm{HH}}=7.4 \mathrm{~Hz}, 3 \mathrm{H} ; \mathrm{CH}_{3}\right) ;{ }^{13} \mathrm{C}\left\{{ }^{1} \mathrm{H}\right\} \mathrm{NMR}\left(150.9 \mathrm{MHz}, \mathrm{CDCl}_{3}\right.$, TMS): $\delta=196.2,168.6,167.9,165.7\left(\mathrm{~d}, J_{\mathrm{CF}}=254.6 \mathrm{~Hz}\right), 158.6,133.4,132.3,130.8(\mathrm{~d}$, $\left.J_{\mathrm{CF}}=9.2 \mathrm{~Hz}\right), 129.2,115.6\left(\mathrm{~d}, J_{\mathrm{CF}}=21.8 \mathrm{~Hz}\right), 113.9,67.2,67.0,57.8,55.1,42.7,40.3$, 21.9, 21.7, 10.3, 10.2; HPLC (Chiracel OD-H, hexane/ $i$-propanol $=19 / 1$, flow rate 0.5 $\mathrm{mL} / \mathrm{min}, \lambda=254 \mathrm{~nm}): \mathrm{t}_{\text {major }}=33.0 \mathrm{~min}, \mathrm{t}_{\mathrm{minor}}=29.4 \mathrm{~min}$, ee $>99 \% ;[\alpha]^{21}{ }_{\mathrm{D}}=+16.28(c=$ 1.0 in $\left.\mathrm{CHCl}_{3}\right)$; ESI-HRMS (m/z) calcd. for $\mathrm{C}_{25} \mathrm{H}_{29} \mathrm{FO}_{6} \mathrm{Na}\left((\mathrm{M}+\mathrm{Na})^{+}\right)$: 467.1846, found: 467.1852 .

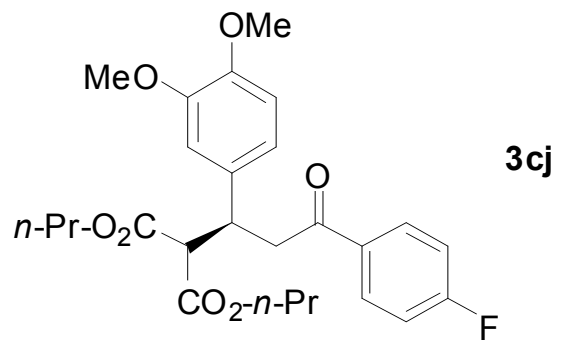

\section{Dipropyl 2-(1-(3,4-dimethoxyphenyl)-3-(4-fluorophenyl)-3-oxopropyl)malonate} (table 3, entry 9): Prepared according to the typical procedure, as described above in $61 \%$ yield. Colorless oil; IR $\left[\mathrm{cm}^{-1}\right]$ (neat): 1728, 1686, 1598, 1519, 1265, 746, 705; ${ }^{1} \mathrm{H}$ NMR (600.2 MHz, $\mathrm{CDCl}_{3}, \mathrm{TMS}$ ): $\delta=7.95-7.91$ (m, 2H; Ar), 7.11-7.06 (m, 2H; Ar), 6.79-6.72 (m, 3H; Ar), 4.16-4.05 (m, 3H; CH, $\left.\mathrm{OCH}_{2}\right), 3.88\left(\mathrm{t},{ }^{3} J_{\mathrm{HH}}=6.6 \mathrm{~Hz}, 2 \mathrm{H} ; \mathrm{OCH}_{2}\right), 3.84(\mathrm{~d}$, 
$\left.{ }^{3} J_{\mathrm{HH}}=9.6 \mathrm{~Hz}, 1 \mathrm{H} ; \mathrm{CH}\right), 3.82\left(\mathrm{~s}, 3 \mathrm{H} ; \mathrm{OCH}_{3}\right), 3.80\left(\mathrm{~s}, 3 \mathrm{H} ; \mathrm{OCH}_{3}\right), \mathrm{ABM}$ spin system $(\mathrm{A}=$ $\left.\mathrm{B}=\mathrm{M}=\mathrm{H}, \delta_{\mathrm{A}}=3.50, \delta_{\mathrm{B}}=3.39,{ }^{2} J_{\mathrm{AB}}=16.3,{ }^{3} J_{\mathrm{AM}}=4.3,{ }^{3} J_{\mathrm{BM}}=9.4 \mathrm{~Hz}, 2 \mathrm{H} ; \mathrm{CH}_{2}\right), 1.64$ (appearance of sext, $\left.{ }^{3} J_{\mathrm{HH}}=7.1 \mathrm{~Hz}, 2 \mathrm{H} ; \mathrm{CH}_{2} \mathrm{CH}_{3}\right), 1.51-1.41\left(\mathrm{~m}, 2 \mathrm{H} ; \mathrm{CH}_{2} \mathrm{CH}_{3}\right), 0.91(\mathrm{t}$, $\left.{ }^{3} J_{\mathrm{HH}}=7.4 \mathrm{~Hz}, 3 \mathrm{H} ; \mathrm{CH}_{3}\right), 0.80\left(\mathrm{t},{ }^{3} J_{\mathrm{HH}}=7.4 \mathrm{~Hz}, 3 \mathrm{H} ; \mathrm{CH}_{3}\right) ;{ }^{13} \mathrm{C}\left\{{ }^{1} \mathrm{H}\right\} \mathrm{NMR}(150.9 \mathrm{MHz}$, $\left.\mathrm{CDCl}_{3}, \mathrm{TMS}\right): \delta=196.3,168.5,167.9,165.7\left(\mathrm{~d}, J_{\mathrm{CF}}=254.8 \mathrm{~Hz}\right), 148.7,148.1,133.4$, $132.9,130.8\left(\mathrm{~d}, J_{\mathrm{CF}}=9.2 \mathrm{~Hz}\right), 120.0,115.6\left(\mathrm{~d}, J_{\mathrm{CF}}=21.8 \mathrm{~Hz}\right), 111.9,111.2,67.2,67.0$, 57.7, 55.9, 55.8, 42.7, 40.7, 21.9, 21.7, 10.3, 10.2; HPLC (Chiralpak AS-H, hexane/ipropanol $=9 / 1$, flow rate $1.0 \mathrm{~mL} / \mathrm{min}, \lambda=254 \mathrm{~nm}$ ): $\mathrm{t}_{\text {major }}=16.4 \mathrm{~min}, \mathrm{t}_{\text {minor }}=14.0 \mathrm{~min}$, ee $=96 \% ;[\alpha]^{22}=+17.02\left(c=0.8\right.$ in $\left.\mathrm{CHCl}_{3}\right)$; ESI-HRMS $(\mathrm{m} / \mathrm{z})$ calcd. for $\mathrm{C}_{26} \mathrm{H}_{31} \mathrm{FO}_{7} \mathrm{Na}$ $\left((\mathrm{M}+\mathrm{Na})^{+}\right):$497.1951, found: 497.1966 .

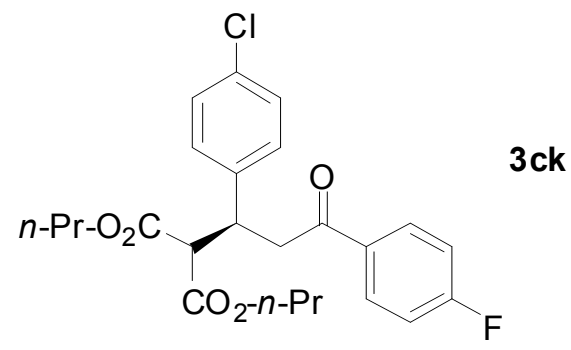

Dipropyl 2-(1-(4-chlorophenyl)-3-(4-fluorophenyl)-3-oxopropyl)malonate (table 3, entry 10): Prepared according to the typical procedure, as described above in $97 \%$ yield. White solid, Mp 69-71 ${ }^{\circ} \mathrm{C}$; IR $\left[\mathrm{cm}^{-1}\right](\mathrm{KBr}): 1730,1685,1600,1300,1238,1157 ;{ }^{1} \mathrm{H}$ NMR (600.2 MHz, $\mathrm{CDCl}_{3}$, TMS): $\delta=7.94-7.90$ (m, 2H; Ar), 7.21 (br s, 4H; Ar), 7.117.07 (m, 2H; Ar), 4.17-4.05 (m, 3H; CH, OCH $), 3.88\left(\mathrm{t},{ }^{3} J_{\mathrm{HH}}=6.7 \mathrm{~Hz}, 2 \mathrm{H} ; \mathrm{OCH}_{2}\right), 3.82$ $\left(\mathrm{d},{ }^{3} J_{\mathrm{HH}}=9.6 \mathrm{~Hz}, 1 \mathrm{H} ; \mathrm{CH}\right), \mathrm{ABM} \operatorname{spin} \operatorname{system}\left(\mathrm{A}=\mathrm{B}=\mathrm{M}=\mathrm{H}, \delta_{\mathrm{A}}=3.53, \delta_{\mathrm{B}}=3.41,{ }^{2} J_{\mathrm{AB}}\right.$ $=16.6,{ }^{3} J_{\mathrm{AM}}=4.2,{ }^{3} J_{\mathrm{BM}}=9.6 \mathrm{~Hz}, 2 \mathrm{H} ; \mathrm{CH}_{2}$ ), 1.64 (appearance of sext, ${ }^{3} J_{\mathrm{HH}}=7.1 \mathrm{~Hz}, 2 \mathrm{H}$; $\left.\mathrm{CH}_{2} \mathrm{CH}_{3}\right), 1.51-1.41\left(\mathrm{~m}, 2 \mathrm{H} ; \mathrm{CH}_{2} \mathrm{CH}_{3}\right), 0.91\left(\mathrm{t},{ }^{3} J_{\mathrm{HH}}=7.3 \mathrm{~Hz}, 3 \mathrm{H} ; \mathrm{CH}_{3}\right), 0.78\left(\mathrm{t},{ }^{3} J_{\mathrm{HH}}=\right.$ $\left.7.5 \mathrm{~Hz}, 3 \mathrm{H} ; \mathrm{CH}_{3}\right) ;{ }^{13} \mathrm{C}\left\{{ }^{1} \mathrm{H}\right\}$ NMR (150.9 MHz, $\left.\mathrm{CDCl}_{3}, \mathrm{TMS}\right): \delta=195.7,168.3,167.7$, $165.8\left(\mathrm{~d}, J_{\mathrm{CF}}=255.0 \mathrm{~Hz}\right), 139.0,133.2,133.0,130.8\left(\mathrm{~d}, J_{\mathrm{CF}}=9.1 \mathrm{~Hz}\right), 129.7,128.6$, $115.7\left(\mathrm{~d}, J_{\mathrm{CF}}=21.9 \mathrm{~Hz}\right), 67.3,67.1,57.3,42.3,21.8,21.7,10.2,10.2$; HPLC (Chiracel OD-H, hexane $/ i$-propanol $=19 / 1$, flow rate $0.5 \mathrm{~mL} / \mathrm{min}, \lambda=254 \mathrm{~nm}$ ): $\mathrm{t}_{\text {major }}=22.8 \mathrm{~min}$, $\mathrm{t}_{\text {minor }}=20.1 \mathrm{~min}$, ee $=97 \% ;[\alpha]_{\mathrm{D}}^{20}=+20.30\left(c=1.0 \mathrm{in} \mathrm{CHCl}_{3}\right)$; ESI-HRMS $(\mathrm{m} / \mathrm{z})$ calcd. for $\mathrm{C}_{24} \mathrm{H}_{26} \mathrm{ClFO}_{5} \mathrm{Na}\left((\mathrm{M}+\mathrm{Na})^{+}\right)$: 471.1350, found: 471.1350 . 


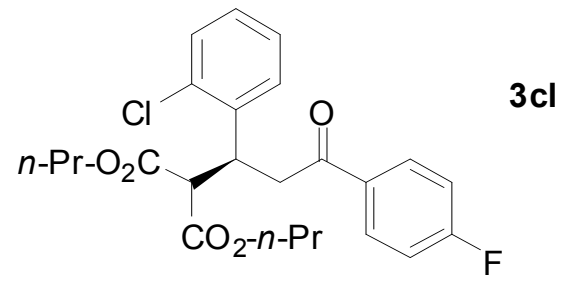

Dipropyl 2-(1-(2-chlorophenyl)-3-(4-fluorophenyl)-3-oxopropyl)malonate (table 3, entry 11): Prepared according to the typical procedure, as described above in $80 \%$ yield. Colorless oil; IR $\left[\mathrm{cm}^{-1}\right]$ (neat): 1732, 1686, 1599, 1265, 1232, 1156, 738, 705; ${ }^{1} \mathrm{H}$ NMR (600.2 MHz, $\left.\mathrm{CDCl}_{3}, \mathrm{TMS}\right): \delta$ = 7.97-7.94 (m, 2H; Ar), 7.34-7.28 (m, 2H; Ar), 7.16-7.06 (m, 4H; Ar), $4.64\left(\mathrm{t} \mathrm{d},{ }^{3} J_{\mathrm{HH}}=5.5,{ }^{3} \mathrm{JHH}_{\mathrm{HH}}=8.3 \mathrm{~Hz}, 1 \mathrm{H} ; \mathrm{CH}\right), 4.12-4.01\left(\mathrm{~m}, 3 \mathrm{H} ; \mathrm{CH}, \mathrm{OCH}_{2}\right.$ ), $3.94\left(\mathrm{t},{ }^{3} \mathrm{~J}_{\mathrm{HH}}=6.6 \mathrm{~Hz}, 2 \mathrm{H} ; \mathrm{OCH}_{2}\right), \mathrm{ABM}$ spin system $\left(\mathrm{A}=\mathrm{B}=\mathrm{M}=\mathrm{H}, \delta_{\mathrm{A}}=3.64, \delta_{\mathrm{B}}=\right.$ $3.60,{ }^{2} J_{\mathrm{AB}}=16.7,{ }^{3} J_{\mathrm{AM}}=8.1,{ }^{3} J_{\mathrm{BM}}=5.1 \mathrm{~Hz}, 2 \mathrm{H} ; \mathrm{CH}_{2}$ ), 1.60 (appearance of sext, ${ }^{3} J_{\mathrm{HH}}=$ $\left.7.1 \mathrm{~Hz}, 2 \mathrm{H} ; \mathrm{CH}_{2} \mathrm{CH}_{3}\right), 1.55-1.45$ (m, $\left.2 \mathrm{H} ; \mathrm{CH}_{2} \mathrm{CH}_{3}\right), 0.87$ (t, ${ }^{3} J_{\mathrm{HH}}=7.3 \mathrm{~Hz}, 3 \mathrm{H} ; \mathrm{CH}_{3}$ ), $0.83\left(\mathrm{t},{ }^{3} J_{\mathrm{HH}}=7.4 \mathrm{~Hz}, 3 \mathrm{H} ; \mathrm{CH}_{3}\right) ;{ }^{13} \mathrm{C}\left\{{ }^{1} \mathrm{H}\right\}$ NMR $\left(150.9 \mathrm{MHz}, \mathrm{CDCl}_{3}, \mathrm{TMS}\right): \delta=196.0$, $168.4,167.9,165.7\left(\mathrm{~d}, J_{\mathrm{CF}}=254.8 \mathrm{~Hz}\right), 137.7,134.1,133.3,130.8\left(\mathrm{~d}, J_{\mathrm{CF}}=9.3 \mathrm{~Hz}\right)$, $130.1,129.3,128.3,126.8,115.6\left(\mathrm{~d}, J_{\mathrm{CF}}=21.8 \mathrm{~Hz}\right), 67.2,67.1,55.2,40.4,37.5,21.8$, 21.7, 10.2, 10.2; HPLC (Chiralpak AD-H, hexane/i-propanol = 9/1, flow rate $1.0 \mathrm{~mL} / \mathrm{min}$, $\lambda=254 \mathrm{~nm}): \mathrm{t}_{\text {major }}=39.9 \mathrm{~min}, \mathrm{t}_{\text {minor }}=16.8 \mathrm{~min}$, ee $=93 \% ;[\alpha]^{22}{ }_{\mathrm{D}}=+35.13(c=1.0 \mathrm{in}$ $\left.\mathrm{CHCl}_{3}\right)$; ESI-HRMS $(\mathrm{m} / \mathrm{z})$ calcd. for $\mathrm{C}_{24} \mathrm{H}_{26} \mathrm{ClFO}_{5} \mathrm{Na}\left((\mathrm{M}+\mathrm{Na})^{+}\right): 471.1350$, found: 471.1335 .

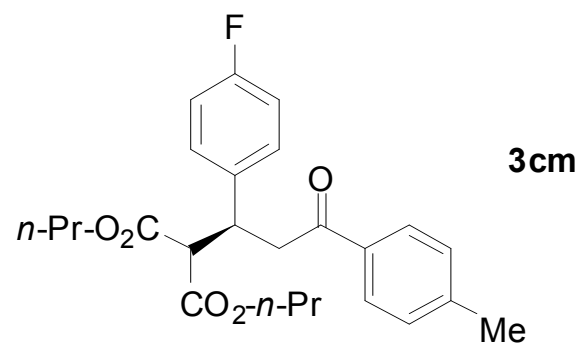

Dipropyl 2-(1-(4-fluorophenyl)-3-oxo-3-p-tolylpropyl)malonate (table 3, entry 12): Prepared according to the typical procedure, as described above in $90 \%$ yield. White solid, Mp 64-67 ${ }^{\circ} \mathrm{C}$; IR $\left[\mathrm{cm}^{-1}\right.$ ] (KBr): 1727, 1673, 1606, 1513, 1296, 1242, 836; ${ }^{1} \mathrm{H}$ NMR (600.2 MHz, $\left.\mathrm{CDCl}_{3}, \mathrm{TMS}\right): \delta=7.78\left(\mathrm{~d},{ }^{3} \mathrm{~J}_{\mathrm{HH}}=8.2 \mathrm{~Hz}, 2 \mathrm{H} ; \mathrm{Ar}\right), 7.27-7.20(\mathrm{~m}, 4 \mathrm{H} ; \mathrm{Ar})$, 6.93-6.89 (m, 2H; Ar), 4.20-4.05 (m, 3H; CH, OCH $), 3.86\left(\mathrm{t},{ }^{3} J_{\mathrm{HH}}=6.6 \mathrm{~Hz}, 2 \mathrm{H} ; \mathrm{OCH}_{2}\right)$, $3.82\left(\mathrm{~d},{ }^{3} J_{\mathrm{HH}}=9.6 \mathrm{~Hz}, 1 \mathrm{H} ; \mathrm{CH}\right), \mathrm{ABM}$ spin system $\left(\mathrm{A}=\mathrm{B}=\mathrm{M}=\mathrm{H}, \delta_{\mathrm{A}}=3.49, \delta_{\mathrm{B}}=3.41\right.$, 
$\left.{ }^{2} J_{\mathrm{AB}}=16.6,{ }^{3} J_{\mathrm{AM}}=4.2,{ }^{3} J_{\mathrm{BM}}=9.5 \mathrm{~Hz}, 2 \mathrm{H} ; \mathrm{CH}_{2}\right), 2.37\left(\mathrm{~s}, 3 \mathrm{H} ; \mathrm{CH}_{3}\right), 1.64$ (appearance of sext, $\left.{ }^{3} \mathrm{~J}_{\mathrm{HH}}=7.1 \mathrm{~Hz}, 2 \mathrm{H} ; \mathrm{CH}_{2} \mathrm{CH}_{3}\right), 1.49-1.40\left(\mathrm{~m}, 2 \mathrm{H} ; \mathrm{CH}_{2} \mathrm{CH}_{3}\right), 0.91\left(\mathrm{t},{ }^{3} J_{\mathrm{HH}}=7.5 \mathrm{~Hz}\right.$, $\left.3 \mathrm{H} ; \mathrm{CH}_{3}\right), 0.79\left(\mathrm{t},{ }^{3} \mathrm{JHH}_{\mathrm{HH}}=7.4 \mathrm{~Hz}, 3 \mathrm{H} ; \mathrm{CH}_{3}\right) ;{ }^{13} \mathrm{C}\left\{{ }^{1} \mathrm{H}\right\} \mathrm{NMR}\left(150.9 \mathrm{MHz}, \mathrm{CDCl}_{3}, \mathrm{TMS}\right): \delta$ $=197.0,168.3,167.8,161.8\left(\mathrm{~d}, J_{\mathrm{CF}}=245.3 \mathrm{~Hz}\right), 143.9,136.3,134.3,129.9\left(\mathrm{~d}, J_{\mathrm{CF}}=7.6\right.$ $\mathrm{Hz}), 129.2,128.2,115.2\left(\mathrm{~d}, J_{\mathrm{CF}}=21.3 \mathrm{~Hz}\right), 67.2,67.0,57.6,42.4,40.2,21.8,21.6,21.5$, 10.2, 10.1; HPLC (Chiralpak AS-H, hexane $/ i$-propanol $=9 / 1$, flow rate $0.5 \mathrm{~mL} / \mathrm{min}, \lambda=$ $254 \mathrm{~nm}): \mathrm{t}_{\text {major }}=30.8 \mathrm{~min}, \mathrm{t}_{\text {minor }}=33.5 \mathrm{~min}$, ee $=98 \% ;[\alpha]_{\mathrm{D}}^{21}=+24.25(c=1.0 \mathrm{in}$ $\left.\mathrm{CHCl}_{3}\right)$; ESI-HRMS (m/z) calcd. for $\mathrm{C}_{25} \mathrm{H}_{29} \mathrm{FO}_{5} \mathrm{Na}\left((\mathrm{M}+\mathrm{Na})^{+}\right)$: 451.1897, found: 451.1859 .

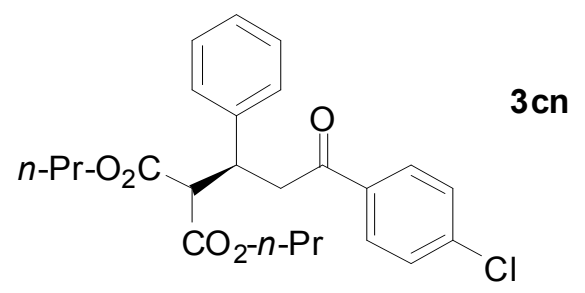

Dipropyl 2-(3-(4-chlorophenyl)-3-oxo-1-phenylpropyl)malonate (table 3, entry 13): Prepared according to the typical procedure, as described above in $98 \%$ yield. Colorless oil; IR $\left[\mathrm{cm}^{-1}\right]$ (neat): 1731, 1687, 1589, 1265, 743, 703; ${ }^{1} \mathrm{H}$ NMR $\left(600.2 \mathrm{MHz}, \mathrm{CDCl}_{3}\right.$, TMS): $\delta=7.84-7.81$ (m, 2H; Ar), 7.39-7.36 (m, 2H; Ar), 7.26-7.21 (m, 4H; Ar), 7.177.14 (m, 1H; Ar), 4.18-4.05 (m, 3H; CH, $\mathrm{OCH}_{2}$ ), 3.87-3.84 (m, 3H; $\mathrm{CH}, \mathrm{OCH}_{2}$ ), ABM spin system $\left(\mathrm{A}=\mathrm{B}=\mathrm{M}=\mathrm{H}, \delta_{\mathrm{A}}=3.53, \delta_{\mathrm{B}}=3.42,{ }^{2} J_{\mathrm{AB}}=16.6,{ }^{3} J_{\mathrm{AM}}=4.4,{ }^{3} J_{\mathrm{BM}}=9.4 \mathrm{~Hz}\right.$, $\left.2 \mathrm{H} ; \mathrm{CH}_{2}\right), 1.63$ (appearance of sext, $\left.{ }^{3} J_{\mathrm{HH}}=7.0 \mathrm{~Hz}, 2 \mathrm{H} ; \mathrm{CH}_{2} \mathrm{CH}_{3}\right), 1.47-1.38(\mathrm{~m}, 2 \mathrm{H}$; $\left.\mathrm{CH}_{2} \mathrm{CH}_{3}\right), 0.90\left(\mathrm{t},{ }^{3} \mathrm{~J}_{\mathrm{HH}}=7.5 \mathrm{~Hz}, 3 \mathrm{H} ; \mathrm{CH}_{3}\right), 0.77\left(\mathrm{t},{ }^{3} J_{\mathrm{HH}}=7.4 \mathrm{~Hz}, 3 \mathrm{H} ; \mathrm{CH}_{3}\right) ;{ }^{13} \mathrm{C}\left\{{ }^{1} \mathrm{H}\right\}$ NMR (150.9 MHz, $\left.\mathrm{CDCl}_{3}, \mathrm{TMS}\right): \delta=196.5,168.5,167.8,140.3,139.4,135.2,129.6$, $128.8,128.5,128.2,127.2,67.2,67.0,57.5,42.6,40.9,21.8,21.6,10.3,10.2 ; \operatorname{HPLC}(2 \mathrm{x}$ Chiracel OJ-H, hexane $/ i$-propanol $=9 / 1$, flow rate $0.5 \mathrm{~mL} / \mathrm{min}, \lambda=254 \mathrm{~nm}$ ): $t_{\text {major }}=76.2$ $\min , \mathrm{t}_{\text {minor }}=68.8 \mathrm{~min}$, ee $=99 \% ;[\alpha]^{21}{ }_{\mathrm{D}}=+17.53\left(c=1.0\right.$ in $\left.\mathrm{CHCl}_{3}\right)$; ESI-HRMS $(\mathrm{m} / \mathrm{z})$ calcd. for $\mathrm{C}_{24} \mathrm{H}_{27} \mathrm{ClO}_{5} \mathrm{Na}\left((\mathrm{M}+\mathrm{Na})^{+}\right)$: 453.1445 , found: 453.1418 . 


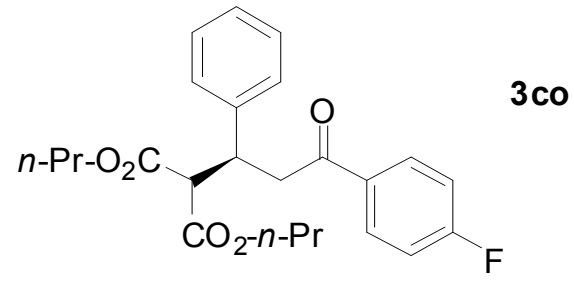

Dipropyl 2-(3-(4-fluorophenyl)-3-oxo-1-phenylpropyl)malonate (table 3, entry 14): Prepared according to the typical procedure, as described above in $92 \%$ yield. Colorless oil; IR $\left[\mathrm{cm}^{-1}\right.$ ] (neat): 1731, 1686, 1599, 1265, 1157, 745, 703; ${ }^{1} \mathrm{H}$ NMR $(600.2 \mathrm{MHz}$, $\left.\mathrm{CDCl}_{3}, \mathrm{TMS}\right): \delta=7.94-7.90(\mathrm{~m}, 2 \mathrm{H} ; \mathrm{Ar}), 7.27-7.21(\mathrm{~m}, 4 \mathrm{H} ; \mathrm{Ar}), 7.18-7.14(\mathrm{~m}, 1 \mathrm{H} ; \mathrm{Ar})$, 7.09-7.05 (m, 2H; Ar), 4.19-4.05 (m, 3H; CH, $\mathrm{OCH}_{2}$ ), 3.87-3.83 (m, 3H; CH, $\mathrm{OCH}_{2}$ ), ABM spin system $\left(\mathrm{A}=\mathrm{B}=\mathrm{M}=\mathrm{H}, \delta_{\mathrm{A}}=3.53, \delta_{\mathrm{B}}=3.43,{ }^{2} J_{\mathrm{AB}}=16.5,{ }^{3} J_{\mathrm{AM}}=4.3,{ }^{3} J_{\mathrm{BM}}=\right.$ $9.5 \mathrm{~Hz}, 2 \mathrm{H} ; \mathrm{CH}_{2}$ ), 1.63 (appearance of sext, $\left.{ }^{3} J_{\mathrm{HH}}=7.0 \mathrm{~Hz}, 2 \mathrm{H} ; \mathrm{CH}_{2} \mathrm{CH}_{3}\right), 1.47-1.38(\mathrm{~m}$, $\left.2 \mathrm{H} ; \mathrm{CH}_{2} \mathrm{CH}_{3}\right), 0.90\left(\mathrm{t},{ }^{3} J_{\mathrm{HH}}=7.3 \mathrm{~Hz}, 3 \mathrm{H} ; \mathrm{CH}_{3}\right), 0.77\left(\mathrm{t},{ }^{3} J_{\mathrm{HH}}=7.3 \mathrm{~Hz}, 3 \mathrm{H} ; \mathrm{CH}_{3}\right) ;{ }^{13} \mathrm{C}\left\{{ }^{1} \mathrm{H}\right\}$ NMR (150.9 MHz, $\left.\mathrm{CDCl}_{3}, \mathrm{TMS}\right): \delta=196.1,168.5,167.8,165.7\left(\mathrm{~d}, J_{\mathrm{CF}}=254.5 \mathrm{~Hz}\right)$, $140.4,133.3,130.8\left(\mathrm{~d}, J_{\mathrm{CF}}=9.2 \mathrm{~Hz}\right), 128.5,128.2,127.2,115.6\left(\mathrm{~d}, J_{\mathrm{CF}}=21.9 \mathrm{~Hz}\right), 67.2$, 67.0, 57.6, 42.5, 40.9, 21.8, 21.7, 10.3, 10.2; HPLC (Chiracel OJ-H, hexane $/ i$-propanol $=$ $19 / 1$, flow rate $0.5 \mathrm{~mL} / \mathrm{min}, \lambda=254 \mathrm{~nm}$ ): $\mathrm{t}_{\text {major }}=41.0 \mathrm{~min}$, $\mathrm{t}_{\text {minor }}=56.7 \mathrm{~min}$, ee $=99 \%$; $[\alpha]^{22}{ }_{D}=+21.69\left(c=1.0\right.$ in $\left.\mathrm{CHCl}_{3}\right)$; ESI-HRMS $(\mathrm{m} / \mathrm{z})$ calcd. for $\mathrm{C}_{24} \mathrm{H}_{27} \mathrm{FO}_{5} \mathrm{Na}\left((\mathrm{M}+\mathrm{Na})^{+}\right)$: 437.1740, found: 437.1729 .

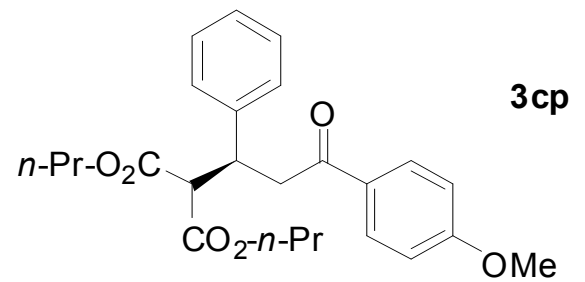

Dipropyl 2-(3-(4-methoxyphenyl)-3-oxo-1-phenylpropyl)malonate (table 3, entry 15): Prepared according to the typical procedure, as described above in $85 \%$ yield. White solid, Mp 63-65 ${ }^{\circ} \mathrm{C}$; IR $\left[\mathrm{cm}^{-1}\right]$ (neat): 1731, 1677, 1602, 1265, 1171, 739, 702; ${ }^{1} \mathrm{H}$ NMR (600.2 MHz, $\left.\mathrm{CDCl}_{3}, \mathrm{TMS}\right): \delta$ = 7.89-7.86 (m, 2H; $\mathrm{Ar}$ ), 7.27-7.20 (m, 4H; Ar), 7.16-7.12 (m, 1H; Ar), 6.89-6.86 (m, 2H; Ar), $4.17\left(\mathrm{t} \mathrm{d},{ }^{3} J_{\mathrm{HH}}=4.6,{ }^{3} J_{\mathrm{HH}}=9.5 \mathrm{~Hz}, 1 \mathrm{H} ; \mathrm{CH}\right), 4.14-$ 4.05 (m, 2H; $\mathrm{OCH}_{2}$ ), 3.87-3.83 (m, 3H; $\left.\mathrm{CH}, \mathrm{OCH}_{2}\right), 3.82\left(\mathrm{~s}, 3 \mathrm{H} ; \mathrm{OCH}_{3}\right), \mathrm{ABM}$ spin system $\left(\mathrm{A}=\mathrm{B}=\mathrm{M}=\mathrm{H}, \delta_{\mathrm{A}}=3.45, \delta_{\mathrm{B}}=3.40,{ }^{2} J_{\mathrm{AB}}=16.3,{ }^{3} J_{\mathrm{AM}}=4.5,{ }^{3} J_{\mathrm{BM}}=9.4 \mathrm{~Hz}, 2 \mathrm{H}\right.$; 
$\mathrm{CH}_{2}$ ), 1.63 (appearance of sext, $\left.{ }^{3} J_{\mathrm{HH}}=7.1 \mathrm{~Hz}, 2 \mathrm{H} ; \mathrm{CH}_{2} \mathrm{CH}_{3}\right), 1.47-1.37(\mathrm{~m}, 2 \mathrm{H}$; $\left.\mathrm{CH}_{2} \mathrm{CH}_{3}\right), 0.90\left(\mathrm{t},{ }^{3} J_{\mathrm{HH}}=7.5 \mathrm{~Hz}, 3 \mathrm{H} ; \mathrm{CH}_{3}\right), 0.77\left(\mathrm{t},{ }^{3} J_{\mathrm{HH}}=7.4 \mathrm{~Hz}, 3 \mathrm{H} ; \mathrm{CH}_{3}\right) ;{ }^{13} \mathrm{C}\left\{{ }^{1} \mathrm{H}\right\}$ NMR (150.9 MHz, $\left.\mathrm{CDCl}_{3}, \mathrm{TMS}\right): \delta=196.1,168.5,167.9,163.4,140.6,130.4,130.0$, $128.4,128.2,127.1,113.7,67.2,66.9,57.7,55.4,42.3,41.0,21.8,21.6,10.3,10.2$; HPLC (Chiracel OD-H, hexane $/ i$-propanol $=19 / 1$, flow rate $0.5 \mathrm{~mL} / \mathrm{min}, \lambda=254 \mathrm{~nm}$ ): $\mathrm{t}_{\text {major }}=40.4 \mathrm{~min}, \mathrm{t}_{\text {minor }}=47.3 \mathrm{~min}$, ee $=99 \% ;[\alpha]_{\mathrm{D}}^{20}=+20.73\left(c=1.0\right.$ in $\left.\mathrm{CHCl}_{3}\right)$; ESIHRMS (m/z) calcd. for $\mathrm{C}_{25} \mathrm{H}_{30} \mathrm{O}_{6} \mathrm{Na}\left((\mathrm{M}+\mathrm{Na})^{+}\right)$: 449.1940, found: 449.1944 .

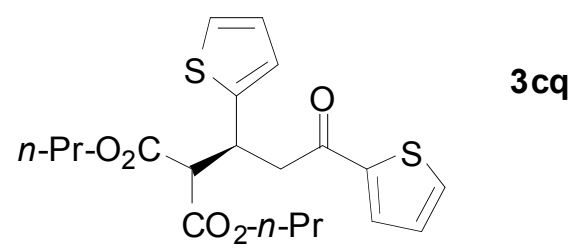

Dipropyl 2-(3-oxo-1,3-di(thiophen-2-yl)propyl)malonate (table 3, entry 16): Prepared according to the typical procedure, as described above in $73 \%$ yield. Beige oil; IR $\left[\mathrm{cm}^{-1}\right]$ (neat): $1734,1663,1416,1265,736,704 ;{ }^{1} \mathrm{H}$ NMR (600.2 MHz, $\left.\mathrm{CDCl}_{3}, \mathrm{TMS}\right): \delta=7.75-$ 7.73 (m, 1H; Ar), 7.61-7.58 (m, 1H; Ar), 7.11-7.08 (m, 2H; Ar), 6.93-6.91 (m, 1H; Ar), 6.86-6.83 (m, 1H; Ar), 4.53-4.48 (m, 1H; CH), 4.14-4.05 (m, 2H; $\left.\mathrm{OCH}_{2}\right), 3.99-3.93(\mathrm{~m}$, $\left.2 \mathrm{H} ; \mathrm{OCH}_{2}\right), 3.90\left(\mathrm{~d},{ }^{3} J_{\mathrm{HH}}=8.4 \mathrm{~Hz}, 1 \mathrm{H} ; \mathrm{CH}\right), 3.48\left(\mathrm{~d},{ }^{3} J_{\mathrm{HH}}=6.8 \mathrm{~Hz}, 2 \mathrm{H} ; \mathrm{CH}_{2}\right), 1.64$ (appearance of sext, ${ }^{3} \mathrm{~J}_{\mathrm{HH}}=7.1 \mathrm{~Hz}, 2 \mathrm{H} ; \mathrm{CH}_{2} \mathrm{CH}_{3}$ ), 1.56-1.48 (m, 2H; $\left.\mathrm{CH}_{2} \mathrm{CH}_{3}\right), 0.91$ (t, $\left.{ }^{3} J_{\mathrm{HH}}=7.3 \mathrm{~Hz}, 3 \mathrm{H} ; \mathrm{CH}_{3}\right), 0.84\left(\mathrm{t},{ }^{3} J_{\mathrm{HH}}=7.3 \mathrm{~Hz}, 3 \mathrm{H} ; \mathrm{CH}_{3}\right) ;{ }^{13} \mathrm{C}\left\{{ }^{1} \mathrm{H}\right\} \mathrm{NMR}(150.9 \mathrm{MHz}$, $\left.\mathrm{CDCl}_{3}, \mathrm{TMS}\right): \delta=190.1,168.1,167.7,144.1,143.3,133.8,132.1,128.1,126.6,125.9$, 124.2, 67.3, 67.2, 57.8, 43.8, 36.3, 21.8, 21.7, 10.3, 10.2; HPLC (Chiralpak AS-H, hexane $/ i$-propanol $=9 / 1$, flow rate $1.0 \mathrm{~mL} / \mathrm{min}, \lambda=254 \mathrm{~nm}$ ): $\mathrm{t}_{\text {major }}=12.7 \mathrm{~min}, \mathrm{t}_{\text {minor }}=$ $17.3 \mathrm{~min}$, ee $=97 \%$; $[\alpha]^{21}{ }_{\mathrm{D}}=+22.21\left(c=1.0\right.$ in $\left.\mathrm{CHCl}_{3}\right)$, ESI-HRMS $(\mathrm{m} / \mathrm{z})$ calcd. for $\mathrm{C}_{20} \mathrm{H}_{24} \mathrm{O}_{5} \mathrm{~S}_{2} \mathrm{Na}\left((\mathrm{M}+\mathrm{Na})^{+}\right)$: 431.0963, found: 431.0922 .

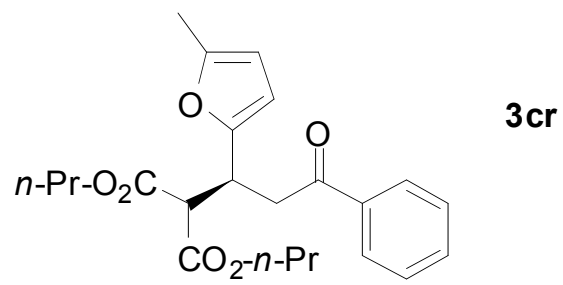


Dipropyl 2-(1-(5-methylfuran-2-yl)-3-oxo-3-phenylpropyl)malonate (table 3, entry 17): Prepared according to the typical procedure, as described above in $71 \%$ yield. Yellow oil; IR $\left[\mathrm{cm}^{-1}\right.$ ] (neat): $1735,1686,1265,750 ;{ }^{1} \mathrm{H}$ NMR $\left(600.2 \mathrm{MHz}, \mathrm{CDCl}_{3}, \mathrm{TMS}\right)$ : $\delta=7.95\left(\mathrm{br} \mathrm{d}, J_{\mathrm{HH}}=7.4 \mathrm{~Hz}, 2 \mathrm{H} ; \mathrm{Ar}\right), 7.58-7.51$ (m, 1H; Ar), 7.47-7.41 (m, 2H; Ar), 5.95 (br s, 1H; Ar), 5.76 (br s, 1H; Ar), 4.29-4.23 (m, 1H; CH), 4.14-3.90 (m, 5H; $\mathrm{CH}, \mathrm{OCH}_{2}$, $\mathrm{OCH}_{2}$ ), 3.58-3.41 (m, 2H; $\mathrm{CH}_{2}$ ), 2.17 (s, 3H, $\left.\mathrm{CH}_{3}\right), 1.68-1.53$ (m, 4H; $\mathrm{CH}_{2} \mathrm{CH}_{3}, \mathrm{CH}_{2} \mathrm{CH}_{3}$ ), 0.95-0.85 (m, 6H; $\left.\mathrm{CH}_{3}, \mathrm{CH}_{3}\right) ;{ }^{13} \mathrm{C}\left\{{ }^{1} \mathrm{H}\right\}$ NMR (150.9 MHz, $\left.\mathrm{CDCl}_{3}, \mathrm{TMS}\right): \delta=197.5$, 168.2, 168.0, 151.6, 151.0, 136.8, 133.1, 128.5, 128.1, 107.7, 106.1, 67.1, 67.1, 55.3, 39.8, 34.4, 21.8, 21.8, 13.5, 10.3, 10.3; HPLC (Chiralpak AD-H, hexane/ $i$-propanol $=19 / 1$, flow rate $0.5 \mathrm{~mL} / \mathrm{min}, \lambda=254 \mathrm{~nm}$ ): $\mathrm{t}_{\text {major }}=40.8 \mathrm{~min}, \mathrm{t}_{\text {minor }}=37.2 \mathrm{~min}$, ee $=96 \% ;[\alpha]^{21} \mathrm{D}$ $=+9.82\left(c=1.0\right.$ in $\left.\mathrm{CHCl}_{3}\right)$; ESI-HRMS $(\mathrm{m} / \mathrm{z})$ calcd. for $\mathrm{C}_{23} \mathrm{H}_{28} \mathrm{O}_{6} \mathrm{Na}\left((\mathrm{M}+\mathrm{Na})^{+}\right)$: 423.1784, found: 423.1777 .

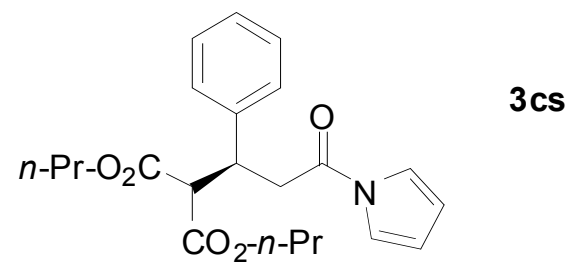

Dipropyl 2-(3-oxo-1-phenyl-3-(1H-pyrrol-1-yl)propyl)malonate (table 3, entry 18): Prepared according to the typical procedure, as described above in $93 \%$ yield. White solid, Mp 88-91 ${ }^{\circ} \mathrm{C}$; IR $\left[\mathrm{cm}^{-1}\right.$ ] (KBr): 1716, 1471, 1280, 1229, 1172, 748; ${ }^{1} \mathrm{H}$ NMR (600.2 $\left.\mathrm{MHz}, \mathrm{CDCl}_{3}, \mathrm{TMS}\right): \delta=7.30-7.25(\mathrm{~m}, 6 \mathrm{H} ; \mathrm{Ar}), 7.22-7.18(\mathrm{~m}, 1 \mathrm{H} ; \mathrm{Ar}), 6.26-6.23(\mathrm{~m}, 2 \mathrm{H}$; Ar), 4.16-4.06 (m, 3H; $\left.\mathrm{CH}, \mathrm{OCH}_{2}\right), 3.88-3.84\left(\mathrm{~m}, 3 \mathrm{H} ; \mathrm{CH}, \mathrm{OCH}_{2}\right), \mathrm{ABM}$ spin system (A $\left.=\mathrm{B}=\mathrm{M}=\mathrm{H}, \delta_{\mathrm{A}}=3.45, \delta_{\mathrm{B}}=3.29,{ }^{2} J_{\mathrm{AB}}=16.3,{ }^{3} J_{\mathrm{AM}}=4.2,{ }^{3} J_{\mathrm{BM}}=9.7 \mathrm{~Hz}, 2 \mathrm{H} ; \mathrm{CH}_{2}\right), 1.64$ (appearance of sext, ${ }^{3} J_{\mathrm{HH}}=7.2 \mathrm{~Hz}, 2 \mathrm{H} ; \mathrm{CH}_{2} \mathrm{CH}_{3}$ ), 1.48-1.39 (m, $\left.2 \mathrm{H} ; \mathrm{CH}_{2} \mathrm{CH}_{3}\right), 0.90$ (t, $\left.{ }^{3} J_{\mathrm{HH}}=7.5 \mathrm{~Hz}, 3 \mathrm{H} ; \mathrm{CH}_{3}\right), 0.78\left(\mathrm{t},{ }^{3} J_{\mathrm{HH}}=7.5 \mathrm{~Hz}, 3 \mathrm{H} ; \mathrm{CH}_{3}\right) ;{ }^{13} \mathrm{C}\left\{{ }^{1} \mathrm{H}\right\} \mathrm{NMR}(150.9 \mathrm{MHz}$, $\left.\mathrm{CDCl}_{3}, \mathrm{TMS}\right): \delta=168.3,168.2,167.6,139.6,128.6,128.1,127.5,119.1,113.1,67.3$, 67.1, 57.2, 41.1, 38.7, 21.8, 21.6, 10.2, 10.2; HPLC (Chiralpak AS-H, hexane/i-propanol $=19 / 1$, flow rate $0.5 \mathrm{~mL} / \mathrm{min}, \lambda=254 \mathrm{~nm}$ ): $\mathrm{t}_{\text {major }}=24.4 \mathrm{~min}, \mathrm{t}_{\text {minor }}=31.2 \mathrm{~min}$, ee $=99 \%$; $[\alpha]_{D}^{20}=+16.38\left(c=1.0\right.$ in $\left.\mathrm{CHCl}_{3}\right)$; ESI-HRMS $(\mathrm{m} / \mathrm{z})$ calcd. for $\mathrm{C}_{22} \mathrm{H}_{27} \mathrm{NO}_{5} \mathrm{Na}$ $\left((\mathrm{M}+\mathrm{Na})^{+}\right): 408.1787$, found: 408.1755 . 


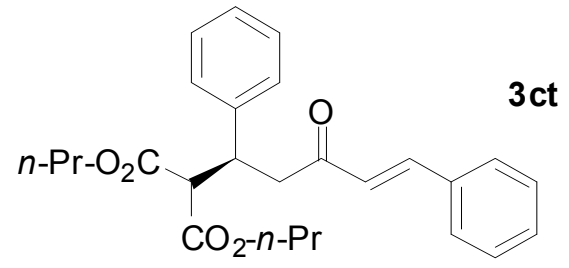

Dipropyl 2-(3-oxo-1,5-diphenylpent-4-enyl)malonate (table 3, entry 19): Prepared according to the typical procedure, as described above in $75 \%$ yield $(97 \%$ yield when

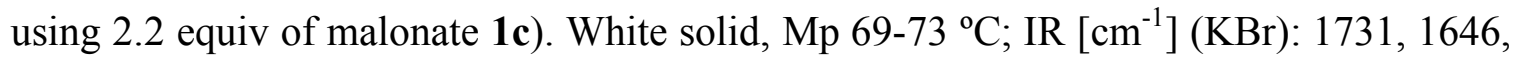
1227, 1163, 705; ${ }^{1} \mathrm{H}$ NMR (600.2 $\mathrm{MHz} \mathrm{CDCl}_{3}$, TMS): $\delta=7.51-7.46$ (m, 3H; Ar), 7.377.34 (m, 3H; Ar), 7.29-7.23 (m, 4H; Ar), 7.19-7.14 (m, 1H; Ar), 7.14 (d, ${ }^{3} J_{\mathrm{HH}}=16.4,1 \mathrm{H}$; Ar), 4.16-4.07 (m, 3H; CH, $\left.\mathrm{OCH}_{2}\right)$, 3.87-3.81 (m, 3H; $\left.\mathrm{CH}, \mathrm{OCH}_{2}\right), \mathrm{ABM}$ spin system (A $\left.=\mathrm{B}=\mathrm{M}=\mathrm{H}, \delta_{\mathrm{A}}=3.20, \delta_{\mathrm{B}}=3.16,{ }^{2} J_{\mathrm{AB}}=16.1,{ }^{3} J_{\mathrm{AM}}=4.6,{ }^{3} J_{\mathrm{BM}}=9.2 \mathrm{~Hz}, 2 \mathrm{H} ; \mathrm{CH}_{2}\right), 1.65$ (appearance of sext, $\left.{ }^{3} J_{\mathrm{HH}}=7.1 \mathrm{~Hz}, 2 \mathrm{H} ; \mathrm{CH}_{2} \mathrm{CH}_{3}\right), 1.48-1.38\left(\mathrm{~m}, 2 \mathrm{H} ; \mathrm{CH}_{2} \mathrm{CH}_{3}\right), 0.92(\mathrm{t}$, $\left.{ }^{3} J_{\mathrm{HH}}=7.5 \mathrm{~Hz}, 3 \mathrm{H} ; \mathrm{CH}_{3}\right), 0.77\left(\mathrm{t},{ }^{3} J_{\mathrm{HH}}=7.3 \mathrm{~Hz}, 3 \mathrm{H} ; \mathrm{CH}_{3}\right) ;{ }^{13} \mathrm{C}\left\{{ }^{1} \mathrm{H}\right\} \mathrm{NMR}(150.9 \mathrm{MHz}$, $\left.\mathrm{CDCl}_{3}, \mathrm{TMS}\right): \delta=197.4,168.4,167.8,142.8,140.4,134.5,130.4,128.9,128.4,128.3$, 128.2, 127.2, 126.0, 67.2, 66.9, 57.6, 44.8, 41.0, 21.9, 21.6, 10.3, 10.2; HPLC (Chiralpak AS-H, hexane $/ i$-propanol $=9 / 1$, flow rate $1.0 \mathrm{~mL} / \mathrm{min}, \lambda=254 \mathrm{~nm}): \mathrm{t}_{\text {major }}=11.8 \mathrm{~min}$, $\mathrm{t}_{\text {minor }}=14.4 \mathrm{~min}$, ee $=86 \% ;[\alpha]_{\mathrm{D}}^{22}=+14.56\left(c=1.0\right.$ in $\left.\mathrm{CHCl}_{3}\right)$; ESI-HRMS $(\mathrm{m} / \mathrm{z})$ calcd. for $\mathrm{C}_{26} \mathrm{H}_{30} \mathrm{O}_{5} \mathrm{Na}\left((\mathrm{M}+\mathrm{Na})^{+}\right)$: 445.1991, found: 445.1975 .

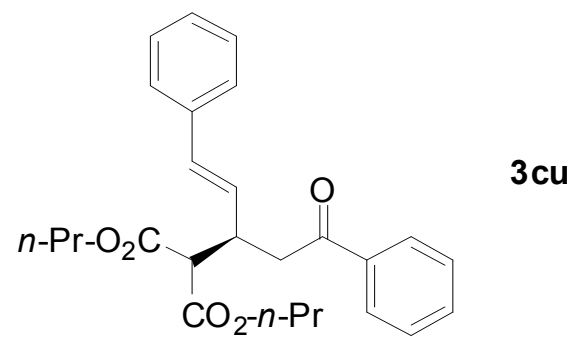

Dipropyl 2-(5-oxo-1,5-diphenylpent-1-en-3-yl)malonate (table 3, entry 20): Prepared according to the typical procedure, as described above in $46 \%$ yield (62\% yield when

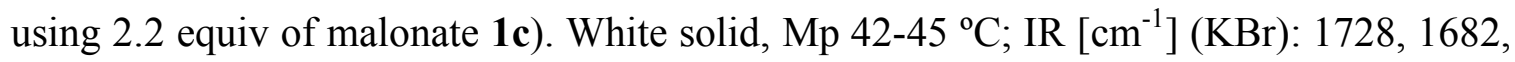
1234, 754, 692; ${ }^{1} \mathrm{H}$ NMR (600.2 MHz, $\mathrm{CDCl}_{3}$, TMS): $\delta=7.98-7.94$ (m, 2H; Ar), 7.567.52 (m, 1H; Ar), 7.47-7.43 (m, 2H; Ar), 7.29-7.22 (m, 4H; Ar), 7.20-7.16 (m, 1H; Ar), $6.46\left(\mathrm{~d},{ }^{3} J_{\mathrm{HH}}=15.8 \mathrm{~Hz}, 1 \mathrm{H} ; \mathrm{Ar}\right), 6.25\left(\mathrm{dd},{ }^{3} J_{\mathrm{HH}}=15.8,{ }^{3} J_{\mathrm{HH}}=9.0 \mathrm{~Hz}, 1 \mathrm{H} ; \mathrm{Ar}\right), 4.15-4.00$ $\left(\mathrm{m}, 4 \mathrm{H} ; \mathrm{OCH}_{2}, \mathrm{OCH}_{2}\right), 3.78\left(\mathrm{~d},{ }^{3} J_{\mathrm{HH}}=7.5 \mathrm{~Hz}, 1 \mathrm{H} ; \mathrm{CH}\right), 3.73-3.67(\mathrm{~m}, 1 \mathrm{H} ; \mathrm{CH}), \mathrm{ABM}$ 
spin system $\left(\mathrm{A}=\mathrm{B}=\mathrm{M}=\mathrm{H}, \delta_{\mathrm{A}}=3.40, \delta_{\mathrm{B}}=3.27,{ }^{2} J_{\mathrm{AB}}=16.8,{ }^{3} J_{\mathrm{AM}}=4.9,{ }^{3} J_{\mathrm{BM}}=7.9 \mathrm{~Hz}\right.$, $2 \mathrm{H} ; \mathrm{CH}_{2}$ ), $1.68-1.55$ (m, 4H; $\left.\mathrm{CH}_{2} \mathrm{CH}_{3}, \mathrm{CH}_{2} \mathrm{CH}_{3}\right), 0.92$ (t, $\left.{ }^{3} J_{\mathrm{HH}}=7.5 \mathrm{~Hz}, 3 \mathrm{H} ; \mathrm{CH}_{3}\right), 0.87$ (t, $\left.{ }^{3} J_{\mathrm{HH}}=7.5 \mathrm{~Hz}, 3 \mathrm{H} ; \mathrm{CH}_{3}\right) ;{ }^{13} \mathrm{C}\left\{{ }^{1} \mathrm{H}\right\}$ NMR (124.5 MHz, $\left.\mathrm{CDCl}_{3}, \mathrm{TMS}\right): \delta=198.0,168.4$, 168.3, 137.0, 136.9, 133.1, 132.6, 128.6, 128.5, 128.4, 128.2, 127.5, 126.4, 67.1, 67.0, 55.7, 41.3, 38.8, 21.9, 21.8, 10.3, 10.3; HPLC (Chiralpak AS-H, hexane/i-propanol $=$ $40 / 1$, flow rate $0.5 \mathrm{~mL} / \mathrm{min}, \lambda=254 \mathrm{~nm}$ ): $t_{\text {major }}=32.5 \mathrm{~min}$, $t_{\text {minor }}=38.0 \mathrm{~min}$, ee $=97 \%$; $[\alpha]^{21}=+1.20\left(c=1.0\right.$ in $\left.\mathrm{CHCl}_{3}\right)$; ESI-HRMS $(\mathrm{m} / \mathrm{z})$ calcd. for $\mathrm{C}_{26} \mathrm{H}_{30} \mathrm{O}_{5} \mathrm{Na}\left((\mathrm{M}+\mathrm{Na})^{+}\right)$: 445.1991, found: $445.1988, \mathrm{C}_{26} \mathrm{H}_{31} \mathrm{O}_{5}\left((\mathrm{M}+\mathrm{H})^{+}\right)$: 423.2171, found: 423.2150 .

\section{References}

6005-8.

(1) Lowenthal, R. E.; Abiko, A.; Masamune, S. Tetrahedron Lett. 1990, 31,

(2) Hilgraf, R.; Pfaltz, A. Adv. Synth. Catal. 2005, 347, 61-77.

(3) Evans, D. A.; Nelson, S. G. J. Am. Chem. Soc. 1997, 119, 6452-6453.

(4) Bhagat, S.; Sharma, R.; Sawant, D. M.; Sharma, L.; Chakraborti, A. K. J. Mol. Catal. A: Chem. 2006, 244, 20-24.

(5) Evans, D. A.; Borg, G.; Scheidt, K. A. Angew. Chem., Int. Ed. 2002, 41, 3188-3191.

(6) Bae, B.-J.; Park, J. T.; Suh, I.-H. J. Organomet. Chem. 2002, 648, 214-219.

(7) Sasai, H.; Arai, T.; Satow, Y.; Houk, K. N.; Shibasaki, M. J. Am. Chem. Soc. 1995, 117, 6194-8.

(8) Kubota, Y.; Ikeya, H.; Sugi, Y.; Yamada, T.; Tatsumi, T. J. Mol. Catal. A: Chem. 2006, 249, 181-190.

(9) Yasuda, K.; Shindo, M.; Koga, K. Tetrahedron Lett. 1996, 37, 6343-6346.

(10) End, N.; Macko, L.; Zehnder, M.; Pfaltz, A. Chem. Eur. J. 1998, 4, 818824.

(11) Wang, J.; Li, H.; Zu, L.; Jiang, W.; Xie, H.; Duan, W.; Wang, W. J. Am. Chem. Soc. 2006, 128, 12652-12653. 